\title{
Ultrastrong, Highly Conductive and Capacitive Hydrogel Electrode for Electron-ion Transduction
}

\section{Bowen Yao}

University of California Los Angeles

Yichen Yan

University of California Los Angeles

Quincy Cui

University of California Los Angeles

Sidi Duan

University of California Los Angeles

Canran Wang

University of California Los Angeles

Yingjie Du

University of California Los Angeles

Yusen Zhao

University of California Los Angeles

Dong Wu

University of California Los Angeles

Shuwang Wu

University of California Los Angeles

Xinyuan Zhu

Shanghai Jiao Tong University

Tzung Hsiai

University of California Los Angeles

Ximin He ( $\nabla$ ximinhe@ucla.edu )

University of California Los Angeles https://orcid.org/0000-0001-8845-4637

Article

Keywords:

Posted Date: January 26th, 2022

DOI: https://doi.org/10.21203/rs.3.rs-1267787/v1 
License: (c) (i) This work is licensed under a Creative Commons Attribution 4.0 International License. Read Full License 


\section{Ultrastrong, Highly Conductive and Capacitive Hydrogel Electrode for Electron-ion}

\section{Transduction}

3 Authors: Bowen Yao ${ }^{1,2 \dagger}$, Yichen Yan ${ }^{1 \dagger}$, Quincy Cui ${ }^{3}$, Sidi Duan ${ }^{1}$, Canran Wang ${ }^{1}$, Yingjie Du ${ }^{1}$, Yusen $\mathrm{Zhao}^{1}$, Dong $\mathrm{Wu}^{1}$, Shuwang, $\mathrm{Wu}^{1,2}$, Xinyuan $\mathrm{Zhu}^{2}$, Tzung Hsiai ${ }^{3}$, Ximin $\mathrm{He}^{1^{*}}$

Affiliations: ${ }^{1}$ Department of Materials Science and Engineering, University of California, Los Angeles, CA 90095.

${ }^{2}$ School of Chemistry and Chemical Engineering, State Key Laboratory of Metal Matrix Composites, Shanghai Jiao Tong University, Shanghai 200240, China. †These authors contributed equally to this work.

\section{Abstract}

Electron-ion transduction is the cornerstone for electronic devices interfacing with biological organisms and promoting emerging ionotronic devices, ranging from basic electronic elements to wearable electronics and energy harvesting devices. However, with commonly used metal electrodes, the electron-ion transduction suffers from high impedance, signal distortion and poor voltage tolerance. Surface modification with conductive porous materials could partially remedy these issues but is inevitably accompanied by weak interfacial adhesion and mechanical weakness. Therefore, freestanding electrodes integrating high mechanical and electrical properties are highly demanded. Herein, a general strategy is discovered to ameliorate these issues by introducing a conducting polymer (CP) hydrogel electrode of ultrahigh strength ( $30 \mathrm{MPa}$ ) and conductivity (up to $\sim 1200 \mathrm{~S} \mathrm{~cm}^{-1}$ ) with a capacitive behavior. These features are derived from the conductive nanoporous matrix with $\pi-\pi$ interactions as both crosslinking sites and electron-transfer pathways, through surface gelation coupled with secondary-doping and densification. This strategy significantly decreased the low-frequency impedance and improved the signal fidelity, without affecting its high-frequency response. Furthermore, excellent biocompatibility, multifunctionality, and heart pacing upon ultra-low voltage $(60 \%$ reduction) have also been demonstrated, showing the great potential of this $\mathrm{CP}$ material for bioelectronic applications and various human-machine interfaces. 
While living organisms transmit signals via ions ${ }^{1}$, conventional manmade electronic devices mostly rely on electron transport. To bridge the electronic realm and the biological world, two promising technologies - bioelectronics and ionotronics - have emerged, attempting to transduce electronic and ionic signals in both directions. For example, bioelectronics is developed to detect electrophysiological signals derived from the ionic concentration gradience across cell membranes, including electrocardiogram (ECG) for the diagnosis of heart disease ${ }^{2}$, electroencephalography (EEG) for brain-computer interface ${ }^{3}$. Reversely, bioelectronics can also deliver ions into bio-tissues for medical therapy ${ }^{4,5}$, typically in electrical stimulation as brain, heart, or muscle disease therapies ${ }^{6,7}$. Meanwhile, ionotronic devices are being developed to be next-generation electronics, which are usually constructed using hydrogels and elastomers and function with a hybrid circuit of mobile ions and mobile electrons. They are expected to open up numerous unprecedented opportunities in energy harvesting (triboelectricity ${ }^{8}$, ionic concentration gradient ${ }^{9,10}$ or thermo-gradient generator ${ }^{11}$ ) and a series of stretchable/soft analogs of conventional electronics, such as flexible transistors ${ }^{12}$, logic circuits ${ }^{13}$, displays ${ }^{14}$ and soft robots ${ }^{15,16}$.

Inert metals were often employed as the electrodes of current bioelectronics and ionotronics to transduce electronic and ionic signals ${ }^{17}, 18$. However, they have several issues, including: (i) a high and unstable impedance across the electrode/electrolyte interface, causing a significant loss and distortion of low-frequency signals $(<100 \mathrm{~Hz})$, which are highly important in many applications, such as low-grade energy conversion (e.g., low-frequency vibration of $<5 \mathrm{~Hz})^{8}$, electromechanical transduction (e.g., actuators working at $\left.0.01-100 \mathrm{~Hz}\right)^{19}$, electrophysiological recordings (e.g., neural local-field potential, typically $<100 \mathrm{~Hz}{ }^{20}$; (ii) a high voltage drop across the electrode/electrolyte interface, due to the high impedance upon low frequency, inducing undesirable faradic electrochemical reactions. One strategy to remedy this issue is introducing a faradic process to the electrode (e.g., $\mathrm{Ag} / \mathrm{AgCl})$, through which electrochemical reaction current passes. However, it suffers from low interfacial stability, mutable electrode potential, and potential toxicity, caused by the $\mathrm{Ag}^{+}$released for long-term implantation ${ }^{21,22}$.

An alternative to employing electrochemical reactions is modifying the inert metal electrodes with conductive porous materials, such as porous carbon materials. With the 
increased electrical-double-layer (EDL) capacitance, the interfacial impedance could be decreased $^{7}$. Although EDL-capacitive materials have been heavily developed to pursue a maximum charge storage ability for energy storage applications, their mechanical strength and conductivity were severely compromised, unbeneficial for the interfacial stability and robustness for bio-/iono-electronics. For example, porous graphene ${ }^{23}, 24$ or metal-oxide frameworks $(\mathrm{MOF})^{25}$ with high EDL capacitances usually have poor mechanical properties (e.g., powder state for MOF) and/or low conductivities (1-100 $\left.\mathrm{S} \mathrm{cm}^{-1}\right)$; poly $(3,4-$ ethylenedioxythiophene):polystyrene sulfonate (PEDOT:PSS) hydrogels could possess both good EDL capacitance and good conductivity, but usually have a low mechanical strength (e.g., 0.1-2 $\mathrm{MPa})^{26-29}$. The intrinsic conflict among capacitance, conductivity and mechanical properties severely limits the molecular design of electrode materials, as a large capacitance requires high porosity which inevitably impairs the electrical conductance pathway and structural integrity. Additionally, using rigid inert metals as the current collectors has been found to induce adverse immune responses at the implantation sites, due to the mechanical mismatch between the electronic materials and organisms (e.g., shear-induced inflammation and the consequent formation of glial scar) ${ }^{30}$.

It is worth noting that, rather than a high energy storage capability, the high fidelity of electron-ion interconversion independent of voltage and frequency is the core for bio/ionoelectronics but this vital issue has not been addressed ${ }^{4}$. Therefore, based on our electrical model and analysis (see Supplementary Discussion Section 1), the ideal electrode material for bio/iono-electronics should have simultaneously three key features: an EDL capacitance behavior (without electrochemical reactions), a high electrical conductivity (alleviating the need for metal current collectors) and good mechanical properties (strong and flexible), along with high stability and good biocompatibility.

Forming EDL requires a conductive matrix with a large specific surface area, while both efficient electron transfer and improved mechanical strength require a high continuity with strong molecular interaction. Thus, we propose that a polymer network with interconnected nano-sized pores and dense pore walls in a continuous, highly-oriented network would be an ideal structure for the electrode material that integrates high mechanical strength and high electrical conductivity with a capacitive behavior. Herein, we have developed a new strategy, surface gelation coupled with secondary doping and partial densification, to create conducting 
96 polymer (CP) hydrogels with such a desirable complex nanostructure. Based on PEDOT:PSS

97 as an exemplary model material in this work, our CP hydrogels are ultra-strong (up to 30 MPa

98 fracture strength), highly conductive (up to $\sim 1,200 \mathrm{~S} \mathrm{~cm}^{-1}$ ) and capacitive (see Supplementary

99 Tables $S 1$ and $S 2$ for detailed comparisons), and thus capable of serving as a freestanding 100 electrode to convert ion-electron signals without involving metal current collectors (Fig. 1a).

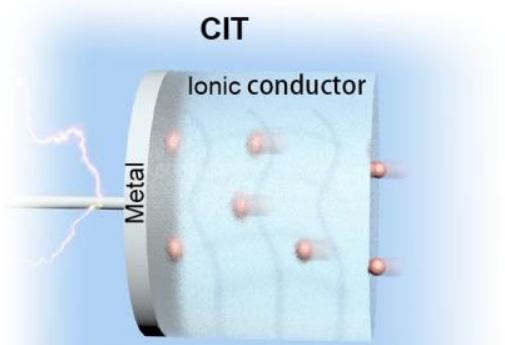

- High impedance \& signal distortion

- Hazardous side reaction

- Rigidity

\section{CPIT}

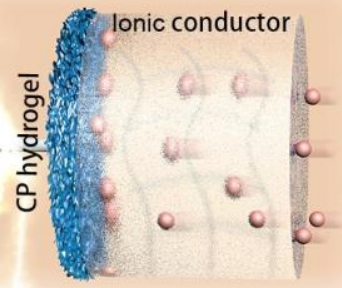

- Low impedance \& high-fidelity signal

- Sustainable operations

- Softness

b

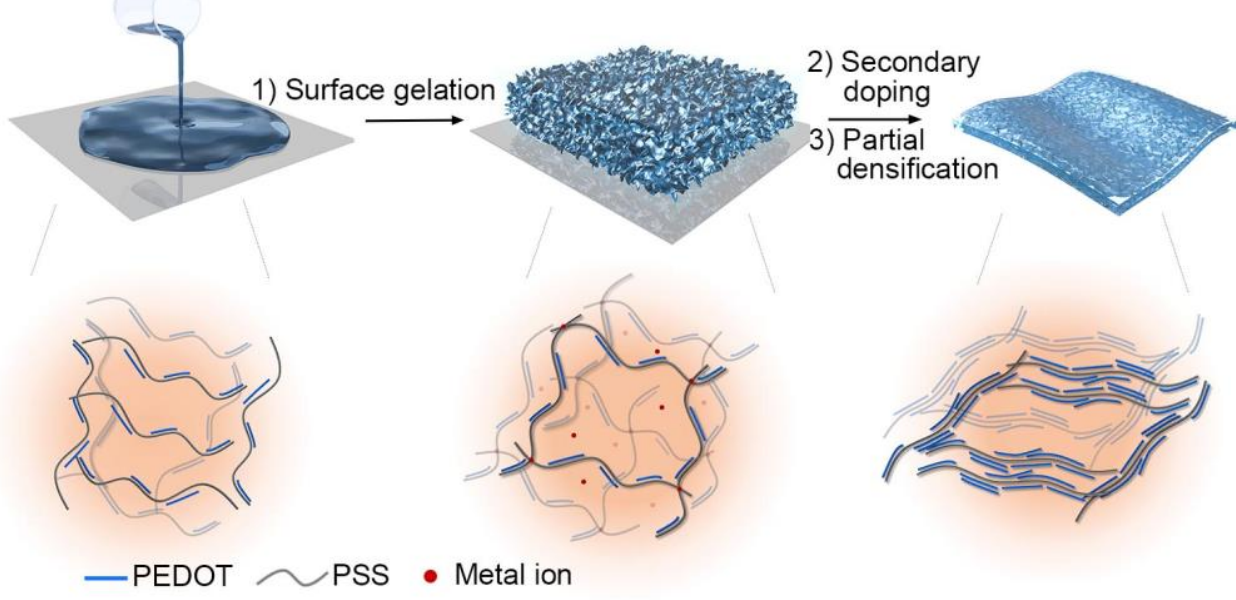

C

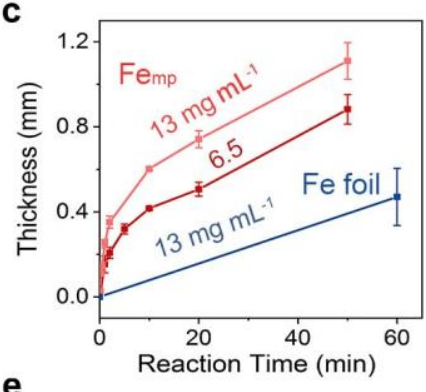

e

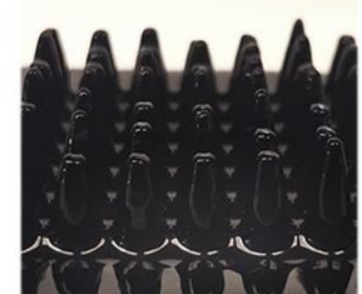

d
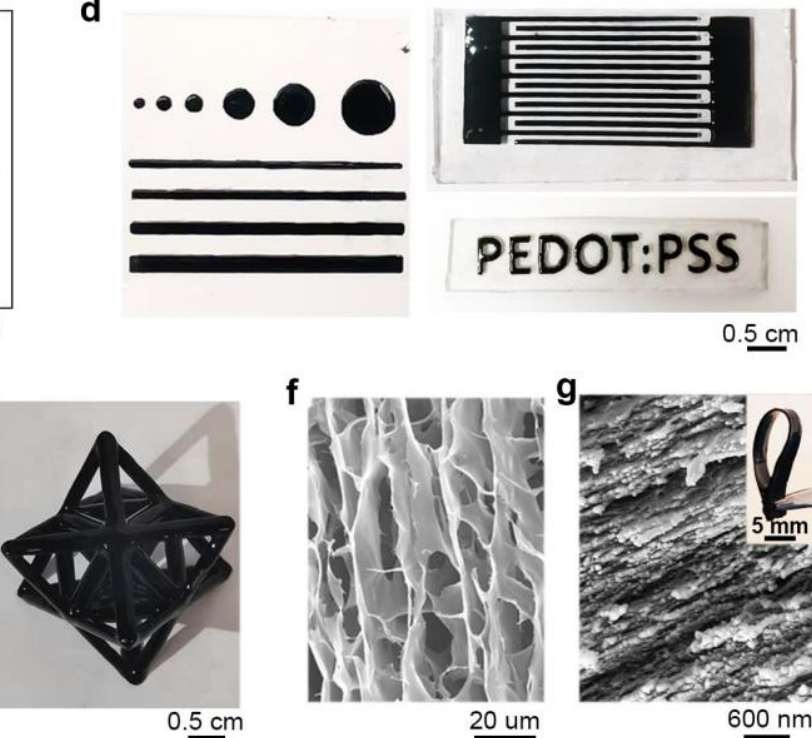

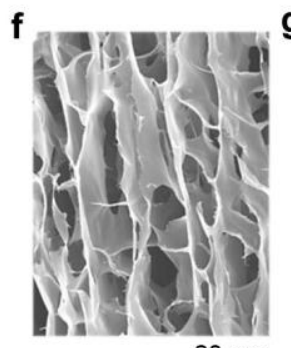

20 um

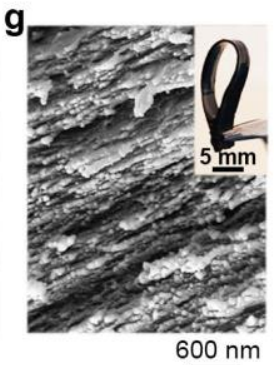



schematics of common ionotronics (CIT) with metal as the electrode and conducting-polymer-assisted ionotronics (CPIT) with PEDOT:PSS hydrogel as the electrode. Compared with CIT, CPIT has a lower interfacial impedance (electrode/electrolyte interface), a lower interfacial voltage for more sustainable operations and a more stable signal transmission ability. b. Preparation procedure of $\mathrm{CP}$ hydrogels with the corresponding structural change of CP chains. The upper panel: CP suspension was applied onto the surface PDMS substrate coated with Fe-microparticle $\left(\mathrm{Fe}_{\mathrm{mp}}\right)$ and kept still for $12 \mathrm{~h}$ for interfacial gelation. Then, a freestanding CP hydrogel film was obtained after secondary doping and partial densification. The lower panel illustrates the transformation of molecular structures of CPs: the CP chains were crosslinked by iron ions that were produced through the in-situ corrosion of Fe powder by acidic PSS chains. Then, after secondary doping and partial densification, the $\mathrm{CP}$ chains underwent a configurational transformation to a laminar structure with a high crystalline degree. $\mathbf{c}$, Gelation kinetics of the $\mathrm{CP}$ suspension on the $\mathrm{Fe}_{\mathrm{mp}}$-coated PDMS or Fe foil. Before acid treatment, the thicknesses of the CP hydrogels, prepared by dropping 13 or $6.5 \mathrm{mg} \mathrm{mL}^{-1} \mathrm{CP}$ suspension on Fe-powder-coated PDMS substrate or Fe foil, were plotted against the reaction time. d, e, Photographs of $\mathrm{CP}$ suspension selectively gelating on the patterned $\mathrm{Fe}_{\mathrm{mp}}$-coated $2 \mathrm{D}$ PDMS substrate (d), 3D PDMS conical pillar array (left in the panel e), and 3D epoxy lattice structure (right in the panel e). f, $\mathbf{g}$, SEM images of freeze-dried CP hydrogel before (f) and after (g) secondary doping.

With these desirable properties, CP-based ionotronics (CPITs) were fabricated to demonstrate their ability of effectively solving the electron-ion transduction issues. By employing the CP hydrogel electrodes, the transduction fidelity was greatly improved with enhanced frequency-independency and voltage-tolerance, through a drastic decrease of the low-frequency impedance at the electrode/ionic-conductor interfaces by nearly two orders of magnitude. Therefore, this strategy may be expected to significantly enhance the performance of various ionotronic devices, including ionic transistors, ionic-driven electric generators and ionic-based bioelectronics. Finally, besides the application in ionotronics, the benefits of CP hydrogel electrodes in bioelectronics were also demonstrated by electrical stimulation promoted cell proliferation and excellent porcine heart pacing with $75 \%$ pacing voltage threshold reduction compared to commercial electrodes, further highlighting the strong ioninjection ability.

\section{Preparation of PEDOT:PSS hydrogels}

The PEDOT:PSS-based CP hydrogel film was synthesized by crosslinking a commercially available CP suspension on a polydimethylsiloxane (PDMS) substrate coated with a 1-3 $\mu \mathrm{m}$ thin layer of carbonyl Fe microparticle $\left(\mathrm{Fe}_{\mathrm{mp}}\right)(\mathrm{Fig}$. 1b, c). The surface gelation mechanism is 
based on the decreased electrostatic repulsion between the negative-charged PEDOT:PSS chains, promoted by the positive-charged iron ions which are uniquely in-situ produced through the corrosion of $\mathrm{Fe}_{\mathrm{mp}}$ by the acidic PSS upon deposition (See Supplementary Discussion Section 2 for detailed mechanism and kinetics studies).

To demonstrate the attractive convenience and versatility of this surface gelation method, $\mathrm{CP}$ hydrogels of various 2D patterns (dot, line, bird, letters, and interdigitation) with a $150 \mu \mathrm{m}$ resolution were fabricated on $\mathrm{Fe}_{\mathrm{mp}}$-coated PDMS simply with shadow masks (Fig. 1d, Supplementary Figs. S2-S4); CP hydrogels grown on the surfaces of complex 3D structures, including lattice, curved surface and needle array, were also constructed (Fig. 1e). Moreover, bulk hydrogels with substantial thicknesses at centimeter-scale could also be prepared, attributed to the good diffusion of metal ions. The entire fabrication could be completed under an ambient condition by employing different active metals besides $\mathrm{Fe}_{\mathrm{mp}}$ as substrates (e.g., $\mathrm{Fe}$, $\mathrm{Zn}, \mathrm{Sn}$, and Al foils) with all off-the-shelf chemicals (Supplementary Figs. S5, S6), demonstrating the easy preparation of the $\mathrm{CP}$ hydrogels and potential for large-scale production. Overall, the surface gelation has a good thickness controllability and benefits conformal coating on various substrates of arbitrary geometries, 2D patterning and 3D printability. Additionally, the resulting CP network contained a large number of nanopores and restricted the excessive stacking of $\mathrm{CP}$ building blocks in the following secondary doping and partial densification steps, thus beneficial to the EDL formation.

Yet, these as-prepared PEDOT:PSS hydrogels $\left(\mathrm{CP}_{\mathrm{ap}}\right)$ with electrostatic attraction-based crosslinking are mechanically weak and possess low conductivity $\left(<1 \mathrm{~S} \mathrm{~cm}^{-1}\right)$, not satisfying the requirements of serving as the electrodes. Fortunately, their electrical and mechanical properties could be greatly improved with a secondary doping method via phase separation and configurational transformation mechanisms ${ }^{42}$. Here, $\mathrm{H}_{2} \mathrm{SO}_{4}$ was used as a treating agent due to its inexpensiveness and outstanding performance (See Supplementary Discussion Section 3 for detailed mechanism studies). Subsequently, the pore structure, another important parameter influencing the properties of PEDOT:PSS hydrogel, was also carefully tuned by a 'controlled densification' strategy, where the hydrogels were infiltrated with a diluted $\mathrm{H}_{2} \mathrm{SO}_{4}$ aqueous solution, dried and then dialyzed in sequence. During this process, the water evaporation drove the shrinkage of micropores into nanopores and also a decrease in the hydrogel membrane thickness, while the majority of original nanopores were well retained (Supplementary Fig. S11). The degree of such structure modifications could be controlled by the water concentration 
in the $\mathrm{H}_{2} \mathrm{SO}_{4}$ solutions. Overall, as a result, the large number of nanopores enabled a high volumetric capacitance, and the densified polymer network (pore wall) effectively further improved the mechanical strength and conductivity, owing to the increased crosslinking

171 densities through more and stronger $\pi-\pi$ interactions and interconnection of the conductive 172 PEDOT-rich domains. Additionally, with the stronger interaction of PEDOT:PSS chains, the 173 hydrogel could maintain the micro-/nano-morphology without swelling when soaking in purified water or physiological saline, which endowed the material excellent structural stability.

175
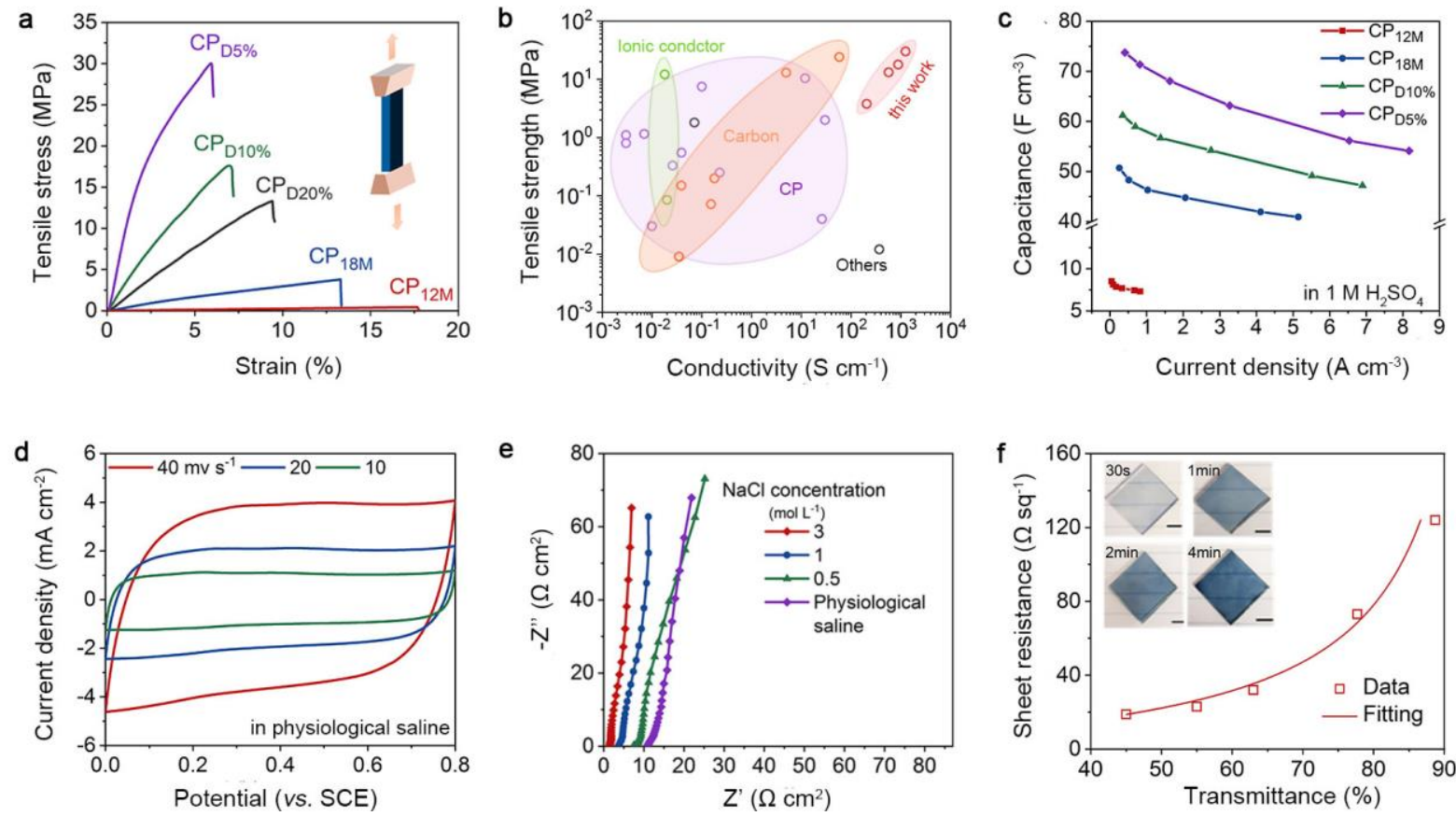

Fig. 2| Properties of the PEDOT:PSS (CP) hydrogels. a, Tensile stress-strain curves of freestanding CP hydrogels at the strain rate of $100 \% \mathrm{~min}^{-1}$. b, An Ashby-style plot comparing the conductivity and strength of the $\mathrm{CP}$ hydrogels with the values of previously reported conductive hydrogels (including CP-based, carbon-based or ionic-conductive hydrogels) (data points are from references mentioned in Supplementary Fig. S12 and Supplementary Table S2). c, Comparisons of the volumetric specific capacitance of CP hydrogels in $1 \mathrm{M} \mathrm{H}_{2} \mathrm{SO}_{4}$ electrolyte., calculated from GCD curves (Supplementary Fig. S13-16). d, CVs of $\mathrm{CP}_{18 \mathrm{M}}$ hydrogel (thickness $=24 \mu \mathrm{m}$ ) at different scan rates and current densities in physiological saline by using a three-electrode setup. e, The Nyquist plot of $\mathrm{CP}_{18 \mathrm{M}}$ hydrogel, tested in a three-electrode setup with physiological saline as the electrolyte, at a frequency range of $\sim 10^{5}-0.026 \mathrm{~Hz}$. f, Sheet-resistance vs. transmittance curve of semi-transparent CP hydrogel membrane on PDMS substrate. Insets are the optical image of $\mathrm{CP}$ hydrogel membrane prepared through interfacial gelation for different time $(0.5,1,2$, and 4 $\mathrm{min}$ ) followed by acid treatment and dialysis. Scale bar $=0.5 \mathrm{~cm}$. 
Using the above method, a series of acid-treated PEDOT:PSS hydrogels with tunable mechanical and electrical properties for different practical applications, including hydrogels only underwent secondary doping process with $12 \mathrm{M}$ or $18 \mathrm{M} \mathrm{H}_{2} \mathrm{SO}_{4}$ (namely $\mathrm{CP}_{12 \mathrm{M}}$ or $\mathrm{CP}_{18 \mathrm{M}}$ ) treatment, and dense hydrogels prepared from $\mathrm{CP}_{18 \mathrm{M}}$ followed by controllable densification, by employing $20 \%, 10 \%$ and $5 \%$ (vacid $\left./ \mathrm{v}_{\text {water }}\right) \mathrm{H}_{2} \mathrm{SO}_{4}$ solutions as densifying agents respectively (namely $\mathrm{CP}_{\mathrm{D} 20 \%}, \mathrm{CP}_{\mathrm{D} 10 \%}$, and $\mathrm{CP}_{\mathrm{D} 5 \%}$ ).

\section{Mechanical and electrical properties}

Taking full advantage of regulatable composition, configuration, crystallinity, and micro/nano-porous morphology by secondary doping and partial densification, CP hydrogel films with tunable mechanical properties were therefore obtained. They showed excellently high fractural strength, which increased with the $\mathrm{H}_{2} \mathrm{SO}_{4}$ concentration (in the secondary doping process) and the densification degree of hydrogel, much higher than those of conductive hydrogels reported previously (Fig. 2a, 2b and Supplementary Fig. S12) 28, 29, 31 . We believe this was caused by the high-degree crystallization of PEDOT chains, stronger molecular interaction and denser laminar structure, which were verified by the morphology and structural characterizations (See Supplementary Discussion Section 3) ${ }^{26,32}$. In detail, the CP $\mathrm{D}_{5 \%}$ displayed the highest tensile strength $(\sim 30 \mathrm{MPa})$ with the shortest fracture strain $(\sim 5.9 \%)$, providing outstanding structural stability for soft electronics. The $\mathrm{CP}_{12 \mathrm{M}}$ possessed the lowest tensile strength of $\sim 0.42 \mathrm{MPa}$ with the largest fracture strain of $\sim 17 \%$. The conductivity of the $\mathrm{CP}$ hydrogels also displayed a positive relationship with $\mathrm{H}_{2} \mathrm{SO}_{4}$ concentration (in the secondary doping process) and densification degree, increasing from $143 \mathrm{~S} \mathrm{~cm}^{-1}$ for $\mathrm{CP}_{12 \mathrm{M}}$ to $1254 \mathrm{~S} \mathrm{~cm}^{-1}$

212 for $\mathrm{CP}_{\mathrm{D} 5 \%}$, which are $10 \sim 10^{3}$ higher than the conductivities of previously reported $\mathrm{CP}$ 213 hydrogels with similar solid contents (Fig. 2b).

Whiling having these excellent mechanical properties and conductivity, the PEDOT:PSS hydrogels also showed a nearly ideal EDL capacitance, large enough for efficient electron-ion transduction when serving as both electrodes and conductors. For comparison, other porous materials explored for supercapacitors or batteries before usually suffered from either low conductance or low mechanical strength (Supplementary Table S1 for detailed comparison).

219 For example, porous reduced graphene oxide showed a conductivity of only $30 \mathrm{~S} \mathrm{~cm}^{-1}$; conductive MOF with EDL capacitance was usually in a powder form. To compare the intrinsic electrochemical properties of $\mathrm{CP}$ hydrogels, highly ionically conductive $1 \mathrm{M} \mathrm{H}_{2} \mathrm{SO}_{4}$ electrolyte 
solution was used as an electrolyte at the beginning, the cyclic voltammetry $(\mathrm{CV})$ curves of all CP hydrogel films showed a quasi-rectangular shape and a linear relationship between current and scan rates ranging from 10 to $400 \mathrm{mV} \mathrm{s}^{-1}$ (Supplementary Figs. S13-S16). According to the galvanostatic charge-discharge tests (GCD) and EIS (Fig. 2c, Supplementary Discussion Section 4.1), the $\mathrm{CP}_{12 \mathrm{M}}$ showed the highest areal capacitance $\left(128 \mathrm{mF} \mathrm{cm}^{-2}\right.$ at $\left.0.62 \mathrm{~mA} \mathrm{~cm}{ }^{-2}\right)$ with the lowest volumetric capacitance $\left(8.5 \mathrm{~F} \mathrm{~cm}^{-3}\right.$ at $\left.0.41 \mathrm{~A} \mathrm{~cm}^{-3}\right)$ among all the PEDOT:PSS hydrogels due to the presence of micron-pores. In contrast, $\mathrm{CP}_{\mathrm{D} 5 \%}$ showed a similar areal capacitance $\left(109 \mathrm{mF} \mathrm{cm}{ }^{-2}\right.$ at $\left.0.62 \mathrm{~mA} \mathrm{~cm}^{-2}\right)$ but a significantly high volumetric capacitance $\left(73.7 \mathrm{~F} \mathrm{~cm}^{-3}\right.$ at $0.41 \mathrm{~A} \mathrm{~cm}^{-3}$ ) brought by its dense micro-morphology (Supplementary Figs. S13-S18). These results indicate the majority of nanopores responsible for the EDL formation were successfully retained after secondary doping and partial densification process.

Interestingly, $\mathrm{CP}$ hydrogel could still show capacitive behavior with a similar capacitance $\left(\sim 110 \mathrm{mF} \mathrm{cm}^{-2}\right.$ at $\left.0.62 \mathrm{~mA} \mathrm{~cm}^{-2}\right)$ in $\mathrm{NaCl}$ solution, such as physiological saline, as proved by the CV, GCD and EIS tests (Fig. 2d, 2e, Supplementary Fig. S19-24 and Supplementary Discussion Section 4.2). The good performance in the low $\mathrm{NaCl}$ concentrations probably was attributed to the PSS chains acting as polymer electrolytes to provide more ions for the formation of EDLs, showing attractive merit for its applications in physiological environments (e.g., as bioelectronics devices for biological interface engineering).

Additionally, by taking advantage of the easy processability of surface gelation, optically transparent or semi-transparent conductive PEDOT:PSS hydrogels with thickness $<100 \mathrm{~nm}$ were also fabricated by conducting interfacial gelating in a diluted $\mathrm{CP}$ suspension. They showed an increased sheet resistance with transmittance increase, fitting well with the theoretical equation (Fig. 2f, Supplementary Fig. S25, See Methods for details). Additionally, electrochemical impedance tests indicated the phase angles were near $0^{\circ}$ within the input frequencies of $10^{5} \sim 1 \mathrm{~Hz}$, revealing its electronically conductive characteristics rather than ionic conductance (Supplementary Fig. S25).

\section{The impedance of ionotronics}

Considering its high conductivity and capacitance with good mechanical properties, $\mathrm{CP}_{18 \mathrm{M}}$ hydrogel, as an example, was then employed as both current collector and electrode to remedy 


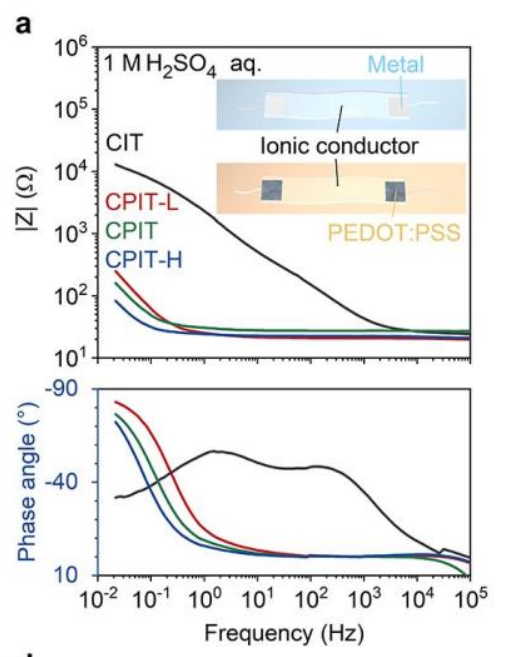

d

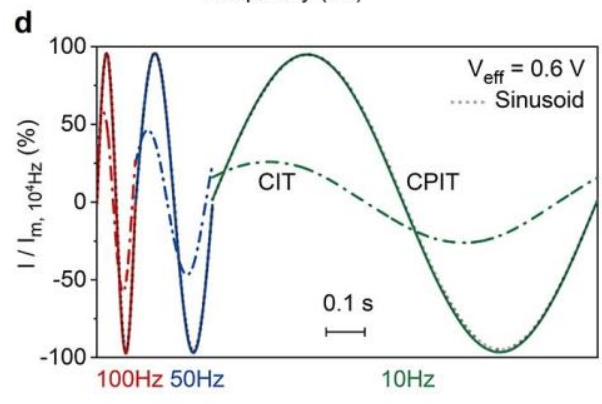

b

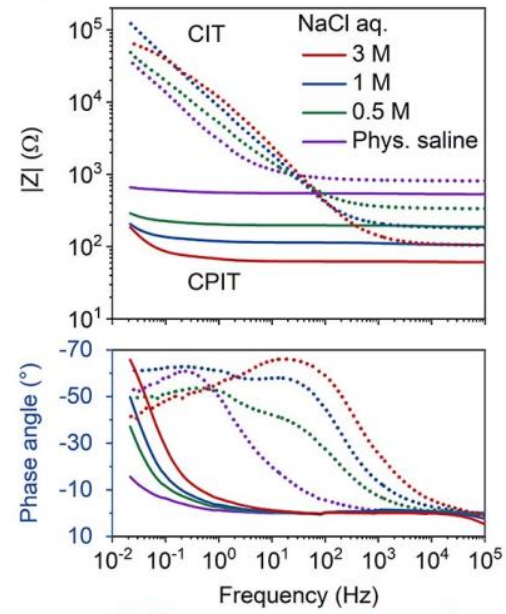

1. Frequency-independent current

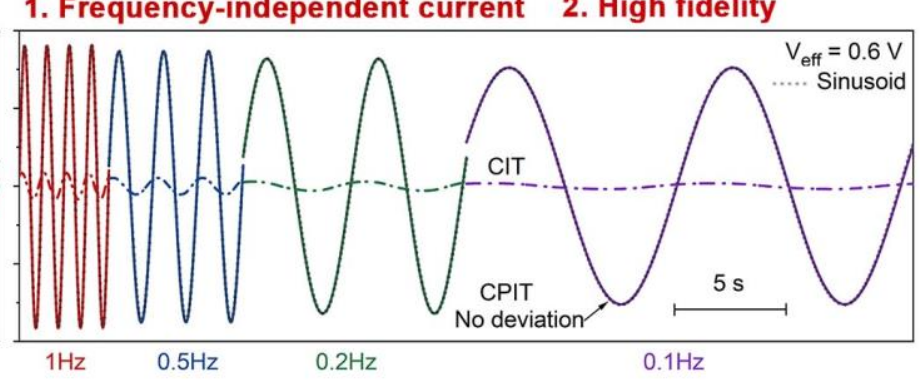

e

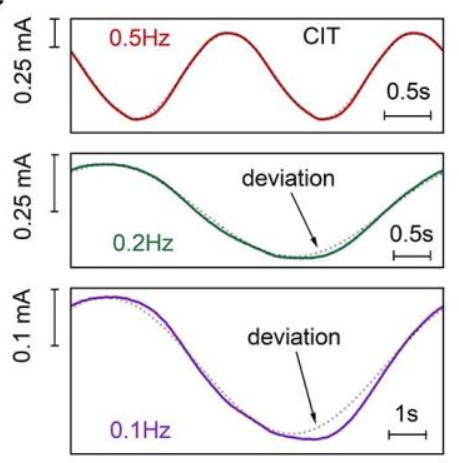

f

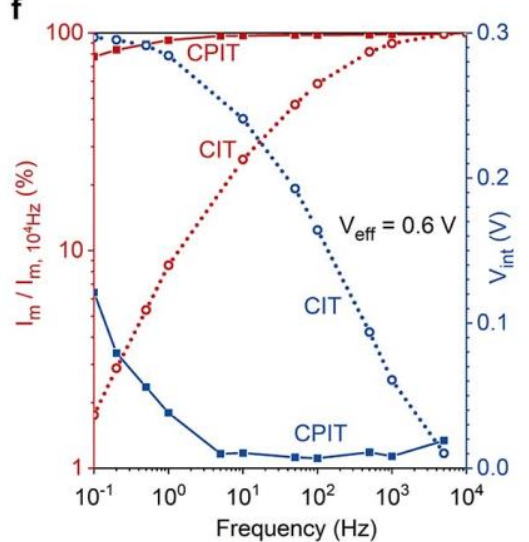

C
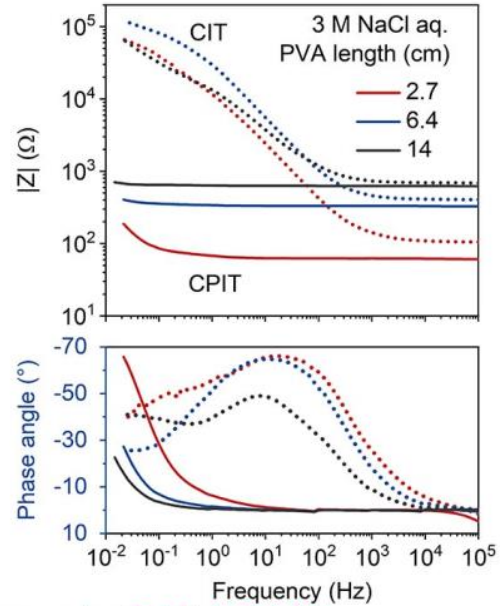

2. High fidelity

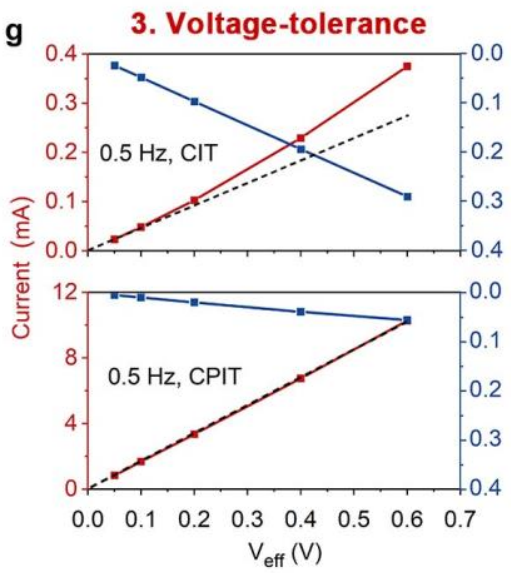

Fig. 3| Electrical properties of common ionotronics (CIT) and conducting polymer-assisted ionotronics

(CPIT). a-c, Bode plots (upper: impedance; lower: phase) of CITs and CPITs with PVA-based ionic conductor, where the CIT counterpart was prepared in the same way as the corresponding CPIT but with Pt plates as current collectors: a, Current collector: $\mathrm{CP}_{18 \mathrm{M}}$ hydrogels of a high, medium and low areal mass loading (CPIT-H, CPIT, CPIT-L, areal capacitance $=250,120,89 \mathrm{mF} \mathrm{cm}^{-2}$ at $0.62 \mathrm{~mA} \mathrm{~cm}{ }^{-2}$, respectively); Electrolyte: $1 \mathrm{M} \mathrm{H}_{2} \mathrm{SO}_{4}$ solution; insets: schematics of CIT (upper) and CPIT (lower). b, Electrolyte: 0.154, $0.5,1$ and $3 \mathrm{M} \mathrm{NaCl}$ solutions; Current collector: $\mathrm{CP}_{18 \mathrm{M}}$ hydrogels of a medium mass loading. $\mathbf{c}$, Ionic conductor: PVA hydrogels of different lengths; Current collector: $\mathrm{CP}_{18 \mathrm{M}}$ of a medium mass loading. $\mathbf{d}$, Normalized current $v s$. time curves of CITs and CPITs upon sinusoidal AC voltage with the efficient voltage ( $\left.\mathrm{V}_{\text {eff }}\right)$ of $0.6 \mathrm{~V}$. For comparison, the currents were normalized with respect to the maximum value of current 
passing through the CIT or CPIT at the AC voltage of $10^{4} \mathrm{~Hz}$. The solid and dashed lines are the normalized current of CPITs and CITs, respectively. The gray dotted lines are fitted sinusoids to clarify the deviation of current-time curves from the sinusoidal waveform. $\mathbf{e}$, The enlarged current-times curves of CITs in $\mathbf{d}$ at lowfrequency voltage. f, Comparisons of the normalized current (red) and the interfacial voltage (blue) of CITs (dotted lines) and CPITs (solid lines) upon sinusoidal voltage input of different frequencies. $\mathbf{g}$, Plots of the current (red) and interfacial voltage (blue) $v s$. $\mathrm{V}_{\text {eff }}$ applied to the CITs and CPITs, upon an input sinusoidal voltage of $0.5 \mathrm{~Hz}$.

269

270 the interfacial issues by reducing the impedance of the electrode/electrolyte interface. First, for comparisons, a CIT (control) was fabricated by infiltrating poly(vinyl alcohol) (PVA) hydrogel with $1 \mathrm{M} \mathrm{H}_{2} \mathrm{SO}_{4}$, which was then attached to two inactive metal $(\mathrm{Pt})$ plates as the electrodes to connect with external circuits (inset in Fig. 3a). According to the Bode impedance plots (Fig. $3 a)$, the CIT showed a low impedance of $\sim 24 \Omega$ with a phase angle of $0.5^{\circ}$ upon AC voltage of $10^{5} \mathrm{~Hz}$, corresponding to the electrolyte resistance of hydrogel $\left(\sim 20 \mathrm{~S} \mathrm{~m}^{-1}\right)$. However, upon the decrease of the alternating signal frequency, the impedance of the CIT saw a quasi-linear rise and finally reached as high as $10^{4} \Omega$, almost 1000 times higher than the value at $10^{5} \mathrm{~Hz}$. Such a high impedance at low frequencies could be mainly attributed to the high impedance of the metal/hydrogel interface. Moreover, in the phase-angle vs. frequency curve (Fig. 3a), upon the decrease of AC frequency, the CIT showed two obvious shoulder peaks at $\sim 100$ and $1 \mathrm{~Hz}$ respectively, with a serious decrease of phase angle when the frequency further decreased to $0.1 \mathrm{~Hz}$, revealing several faradic processes initiated within the metal/hydrogel interface through chemical reactions (possibly derived from the decomposition of electrolyte and/or oxygen reduction reaction). This extremely complex and uncontrolled electrochemical process would severely impact the signal transduction, and possibly cause interfacial instability of the ionic conductor. Overall, the overly high impedance and non-negligible faradic process severely limit the usages of stretchable and transparent ionotronics in the low-frequency-related applications, in the terms of frequency dependence, interfacial stability and electric signal processing.

Alternatively, by replacing the Pt electrode with the $\mathrm{CP}_{18 \mathrm{~m}}$ hydrogel (areal capacitance: $\sim 120 \mathrm{mF} \mathrm{cm}^{-2}$ ) (Fig. 3a, the green line), CPIT showed a CIT-comparable impedance of $\sim 27 \Omega$ upon high-frequency electricity, but its impedance remained stable without significant increment, upon decreasing the frequency from $10^{5}$ to $1 \mathrm{~Hz}$. When the frequency further decreased from 1 to $0.02 \mathrm{~Hz}$, the impedance of EDL gradually dominated and finally reached 
only $\sim 250 \Omega$ at $0.022 \mathrm{~Hz}$. For comparison, the impedance ratio of CIT to CPIT (|Z could reach up to 7, 20, 90, and 120 at the AC frequency of 100, 10, 1 and $0.1 \mathrm{~Hz}$, respectively. Besides, the CPIT also showed a stable phase angle of $\sim 0^{\circ}$ within a wide frequency region with a regular and stable increment under the low-frequency region of $<1 \mathrm{~Hz}$, beneficial for signal transduction and processing. The influence of the complex faradic process on impedance, present in the CIT system, was almost negligible in the CPIT system. This could be ascribed to the low impedance of $\mathrm{CP}$ hydrogel, which increased the overall current and decreased the voltage across the electrode/electrolyte interface to suppress the harmful electrochemical reactions within it. This is consistent with our theoretical analysis (Supplementary Discussion Section 1).

Our proposed CPIT strategy is a general method to ameliorate the low-frequency performance issues for different ionic conductors, which was proved by using $\mathrm{CP}$ hydrogel with different areal capacitances (Fig. 3a), and by using different ionic conductors with different sizes or ionic conductivities (See Supplementary Discussion Section 5 for detailed discussion). For example, similar to the trend observed for the ionotronics with hydrogel infiltrated with $\mathrm{H}_{2} \mathrm{SO}_{4}$ as ionic conductor, all the CPITs with different $\mathrm{NaCl}$ electrolyte concentrations and different types or sizes of hydrogel ionic conductors showed significantly depressed impedance growth at low frequencies with stable phase angles (Fig. 3b, 3c). This allows for taking full advantage of the unique properties of different hydrogels, including softness, stretchability, transparency and biocompatibility, etc., to fabricate ionic conductors of high-performance for different applications.

\section{Current-time measurement, voltage response and electrical loading}

To intuitively study the conductive properties of CIT and CPIT, the current-time curves were recorded when a sinusoidal AC input voltage $(\mathrm{V}(\mathrm{t}))$ was applied $\left(\mathrm{V}(\mathrm{t})=\sqrt{2} \mathrm{~V}_{\text {eff }} \sin (2 \pi \mathrm{ft})\right.$, $V_{\text {eff }}$ is the efficient voltage, $f$ is the frequency). On the whole, the evolved current showed a sinusoidal waveform with a phase difference $\left(\varphi \mathrm{v}-\varphi_{\mathrm{I}}\right)$ dependent on the EDL capacitance and ionic diffusion resistance of the ionic conductor. 

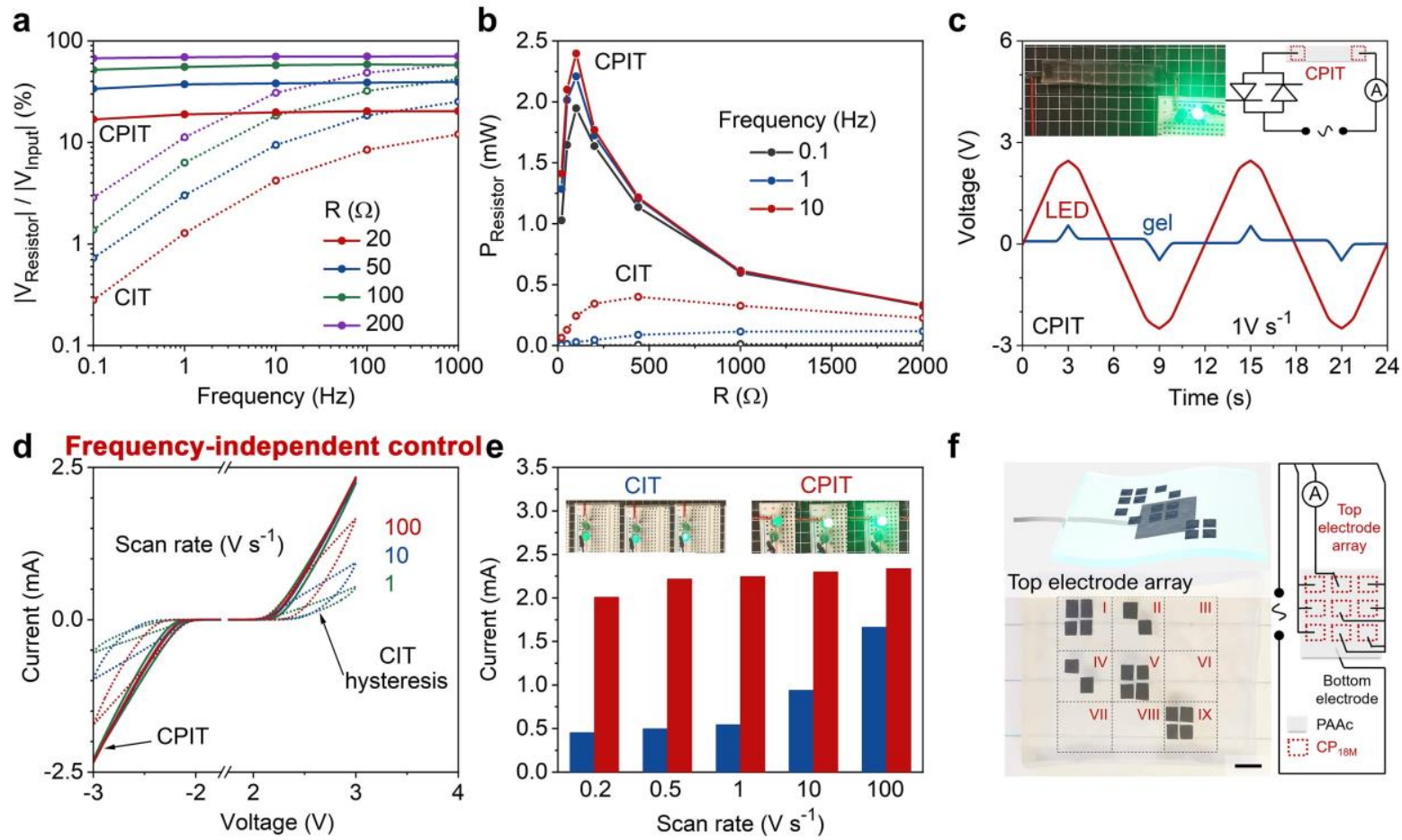

f

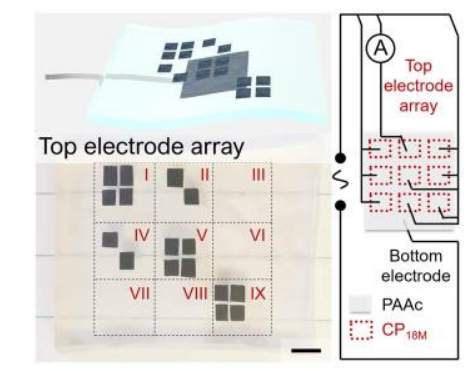

h
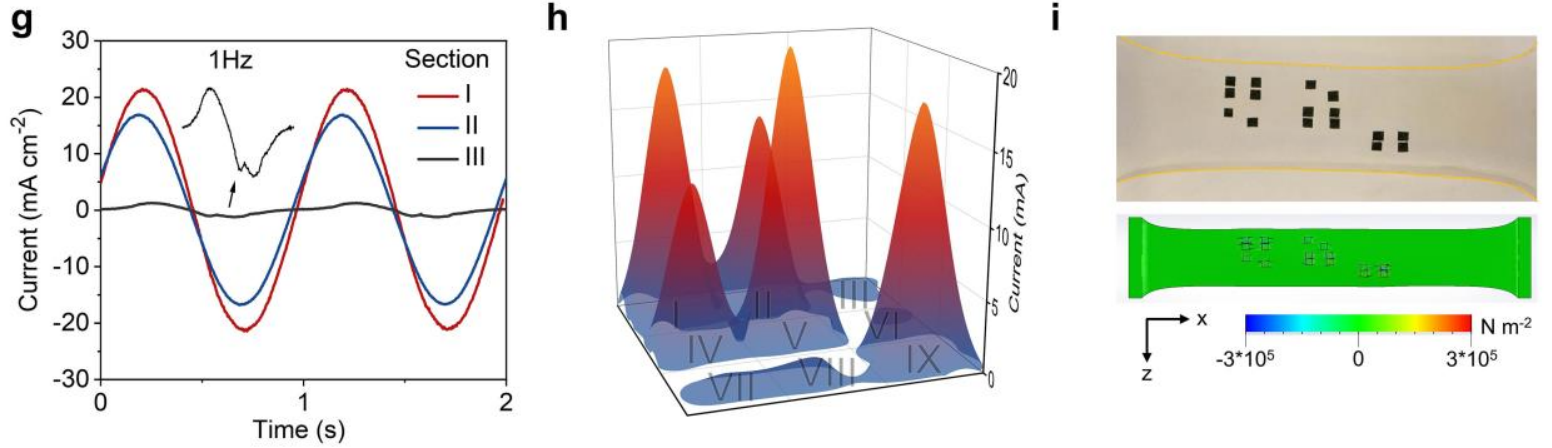

Fig. 4| Demonstrations of the CPIT performance and functions. a, b, Demonstration of CPITs and CITs as ionotronics connected with resistors as electric loadings: a, Voltage ratios of resistors to the peak value of input voltage $\left(\left|\mathrm{V}_{\text {input }}\right|=0.848 \mathrm{~V}\right)$ at different frequencies. $\mathbf{b}$, the output power of the resistors upon $\mathrm{AC}$ voltage of different frequencies. c-e, Demonstration of CPITs and CIT as ionotronics connected with LEDs as electric loadings: two LEDs in parallel with reversed polarity were connected to an AC power source with a triangular waveform: c, Voltage $v s$. time curves of the CPIT and LEDs. Voltage window: $-3 \sim 3$ V. Sweep rate: $1 \mathrm{~V} \mathrm{~s}^{-1}$. Insets: an optical image of the setup and relevant circuit diagram. $\mathbf{d}$, Cyclic voltammogram of the LED circuit based on CITs (dotted lines) or CPITs (solid lines) as ionotronic devices. Scan rate: 1, 10, and $100 \mathrm{~V} \mathrm{~s}^{-1}$. e, Comparison of the whole current passing through LED circuits under AC voltage of different scan rates. Insets: photos of LEDs during their maximum illumination under the input voltage with different scan rates. $\mathbf{f - i}$, Demonstration of an ionotronic device with current density spatial modulation (ICCDSM): f, Schematic diagrams (upper-left) of this ionotronic device where several $\mathrm{CP}_{18 \mathrm{M}}$ hydrogels were attached to one side of an adhesive polyacrylic acid (PAAc) hydrogel as top electrode array, while another $\mathrm{CP}_{18 \mathrm{M}}$ hydrogel on its opposite surface was used as the bottom electrode. The lower-left picture is the digital 
photography of the top electrode. The lower-right picture is the schematic diagram to depict the current measurement: the electrode array which was divided into 9 domains was connected to an AC power through Pt plate, during which the currents passing through each domain was recorded by a current meter. $\mathbf{g}, \mathbf{h}$, Electric response of IC-CDSM upon AC voltage. $\mathrm{V}_{\text {eff: }} 0.6 \mathrm{~V}$; Frequency: $1 \mathrm{~Hz}$. g, Current vs. time curves of the domain I, II, and III on the ionotronic device. Inset: enlarged current profile of domain III. h, Comparisons of peak current densities of different domains. i, Top: the optical image of the stretched ionotronic device. The boundaries of the PAAc hydrogel were marked with yellow lines for visibility. Bottom: the simulation of the shear stress along $\mathrm{x}$-direction in the xz-plane of the stretched IC-CDSM. Black lines indicate the boundaries of the $\mathrm{CP}_{18 \mathrm{M}}$ and the PAAc hydrogel.

Upon AC voltage of high frequency $\left(10^{4} \mathrm{~Hz}\right)$, the CPMIC and CIT shared a comparable efficient current, corresponding to the electrolyte resistance of the ionic conductor, consistent with their impedance results (Supplementary Fig. S34). When the frequency decreased from $10^{5}$ to $0.1 \mathrm{~Hz}$, the current of CIT saw a sharp drop to only $1 \%$ of the original value with a phase difference of $-60^{\circ}$, while the CPMIC remained $90 \%$ of its original current with a phase difference of $-20^{\circ}$ (Fig. 3d-f, Supplementary S34). Additionally, CIT showed an obvious deviation of the current waveform from sinusoidal shapes especially upon low-frequency AC (Fig. 3e). For comparison, the current waveforms through the CPIT were almost identical to a standard sinusoidal regardless of the input frequencies (Fig. 3d), showing the excellent advantages of CPIT as an ionic conductor to perfectly transmit electric signals with high quality. The high deviation in the CIT circuit could be ascribed to the significant electrochemical reaction initiated by the high voltage across the metal/electrolyte interface with a high impedance upon low-frequency AC (Supplementary Discussion Section 1). The interfacial voltage (efficient voltage) can also be directly estimated by vectorially subtracting voltage of electrolyte from input voltage (Fig. 3f, Supplementary Discussion Section 6), which were calculated to be only $\sim 0.015 \mathrm{~V}$ at $10 \mathrm{~Hz}$ and $0.038 \mathrm{~V}$ at $1 \mathrm{~Hz}$ for CPIT upon an input voltage of $0.6 \mathrm{~V}$. They were much less than those of CIT $(\sim 0.24 \mathrm{~V}$ at $10 \mathrm{~Hz}, 0.28 \mathrm{~V}$ at $1 \mathrm{~Hz})$, and consistent with our theoretical analysis.

Furthermore, to demonstrate the CPIT has a better voltage tolerance than CIT, the effect of the amplitude of AC power voltage was also studied. As expected, as the $\mathrm{V}_{\text {eff }}$ increased from $5 \mathrm{mV}$ to $0.6 \mathrm{~V}$, the output current increased linearly for CPIT with a stable phase angle, but nonlinearly for CIT with an unstable phase angle (Fig. 3g, Supplementary Figs. S35 and S36), 
381

382

\section{3}

ascribed to the acceleration of electrochemical reaction at the metal/electrolyte interface of CIT upon high voltages. This phenomenon was further confirmed by the EIS results, where the CIT saw an obvious decrease in impedance and phase angle at the low-frequency region when the amplitude ranged from 0.05 to $0.6 \mathrm{~V}$ (Supplementary Fig. S36). By contrast, with a nearly amplitude-independent impedance and phase angle, the CPIT showed great advantages over CIT in the terms of interfacial stability and electric signal fidelity (Supplementary Fig. S37).

To have a better fundamental understanding of the electric response of ionotronics when an electrical appliance was loaded, a theoretical analysis was therefore conducted first. When loaded with a passive appliance (the equivalent circuit is shown in Supplementary Fig. S1), the efficient current $(|\tilde{I}|)$ flowing through the circuit and the efficient interfacial voltage $\left(\left|\tilde{V}_{E D L}\right|\right)$ across the hydrogel/electrode interface could be expressed by the following equations:

$$
\begin{aligned}
|\tilde{I}| & =\frac{|\tilde{V}|}{\sqrt{\left(2 R_{0}+Z_{A}{ }^{\prime}\right)^{2}+\left(\frac{4}{\omega C_{E D L}}+Z_{A}{ }^{\prime \prime}\right)^{2}}} \\
\left|\tilde{V}_{E D L}\right| & =\frac{|\tilde{V}|}{\sqrt{\left(\omega C_{E D L}\right)^{2}\left(2 R_{0}+Z_{A}{ }^{\prime}\right)^{2}+\left(-4+\omega C_{E D L} Z_{A}{ }^{\prime \prime}\right)^{2}}}
\end{aligned}
$$

, where $\mathrm{Z}_{\mathrm{A}}$ ' and $\mathrm{Z}_{\mathrm{A}}$ "' are the real and imaginary impedances of any passive appliance loaded, $\mathrm{R}_{0}$ and $C_{E D L}$ represent the ionic resistance of hydrogel and EDL capacitance of electrode, and $\omega$ is the angular frequency of AC.

Upon a low-frequency signal, the induction of the appliance can be neglected $\left(\mathrm{Z}_{\mathrm{A}}{ }^{\prime} \leqslant 0\right)$. Therefore, employing electrodes with a much higher $C_{E D L}$ is thus expected to increase the efficient current and lower the voltage applied to the EDL, beneficial for the voltage tolerance and electrochemical stability of ionotronics. By mathematical approximations, when $C_{E D L}$ increases, the current will increase linearly when the loaded appliance has a much smaller impedance than that of EDL in ionotronics $\left(4 / \omega \mathrm{C}_{\mathrm{EDL}}\right)$, or the interfacial voltage will decrease inversely to $\mathrm{C}_{\mathrm{EDL}}$ when a high-impedance appliance is loaded (Supplementary Section 1).

Based on this analysis, we experimentally validated the effectiveness of a highlycapacitive electrode in improving the voltage tolerance and signal fidelity. A CPIT or CIT was connected to the electrical loads (20-2000 $\Omega$ resistors as examples) with an AC power source $\left(\mathrm{V}_{\text {eff }}=0.6 \mathrm{~V}\right)$. The voltage-drop and power of the electrical loads decreased seriously with the 
decrease of the AC frequency for the CIT-based circuit (Fig. 4a, 4b, Supplementary Figs. S38 and S39), but remain excellently stable and high within a wide AC frequency range of 0.11000 for CPIT-based circuit. Then, for a more visualized demonstration, two LEDs with antiparallel polarity were also powered by an AC voltage with a triangular waveform (voltage window: -3 to 3 V) (Fig. 4c, Supplementary Fig. S40 and S41). Overall, the voltage across the LEDs has a nonlinear change with input voltage from 2.3 to $3 \mathrm{~V}$, due to the nonlinear voltagedependent resistance of LED (Fig. 4c, Supplementary Fig. S41) and the non-negligible electrolyte resistance and interfacial impedance of the ionic conductor. According to the current-voltage curves (Figs. 4d and 4e, Supplementary Fig. S41-S47), the CIT-based circuit showed a scan-rate-dependent curves with significant hysteresis and small current (Fig. 4d), indicating the large capacitive impedance of CIT. By contrast, the CPIT-based circuit showed a voltage-dependent current without obvious hysteresis during the scanning cycles, showing the great convenience for LED illumination control. Meanwhile, the voltages across CPIT were much stable $(0.53 \mathrm{~V}$ when the input voltage reached $3 \mathrm{~V})$, almost independent of the scanning rate, while the voltage across CIT showed a strong dependence on scanning rate, and increased significantly from $0.58 \mathrm{~V}$ at a scan rate of $100 \mathrm{~V} \mathrm{~s}^{-1}$ to $0.72 \mathrm{~V}$ at $0.2 \mathrm{~V} \mathrm{~s}^{-1}$ when the input voltage reached $3 \mathrm{~V}$ (Supplementary Fig. S41), indicating that a higher voltage was applied to the interface of CIT, unbeneficial to the structural stability of ionic conductors.

\section{Ionotronics with spatial-resolved current densities}

By taking advantage of the ability of the CP hydrogel in tuning local interfacial impedance, a new soft ionotronic model with the controlled spatial distribution of current density was also demonstrated, which will be highly useful for realizing multi-point selective electrical stimulation with a simple device configuration in neuromulation applications, for example. Here, an exemplary ionotronics model was fabricated by coating a polyacrylic acid (PAAc) hydrogel ionic conductor with patterned $\mathrm{CP}_{18 \mathrm{M}}$ hydrogels (Fig. 4f). For measurements, the ionic conductor was divided into nine sections connected in parallel to an AC power source, and then the current through each was measured in real-time. The spatial distribution of current density can be controlled by the presence (or absence) of the CP hydrogel (Figs. 4g and 4h). As shown in the time-dependent current profiles, upon AC power of $10 \mathrm{~Hz}$, the current densities of the sections with four pieces of $\mathrm{CP}_{18 \mathrm{M}}$ larger current were calculated to be $\sim 25 \mathrm{~mA} \mathrm{~cm}^{-2}$, slightly higher than those with two pieces $\left(\sim 16 \mathrm{~mA} \mathrm{~cm}^{-2}\right)$. By contrast, the section without $\mathrm{CP}$ hydrogel showed only $\sim 10 \%$ of the current density of the sections with four pieces. This demonstrated 


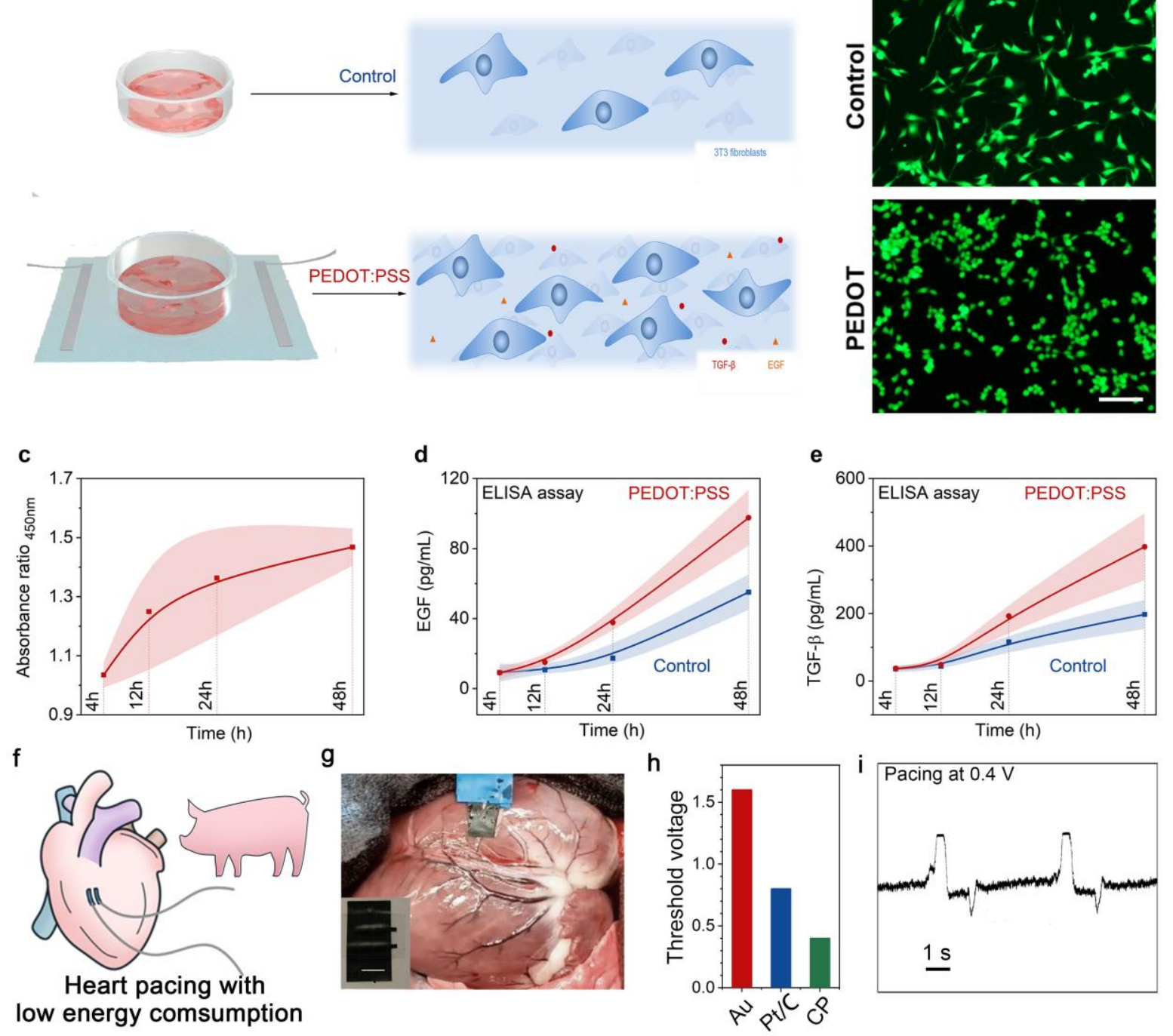

Fig. 5| Biocompatibility of the CP hydrogel. a, Schematics of fibroblasts cultured on a PDMS substrate (control) or a PDMS-supported CP hydrogel substrate with electrical stimulation (CP/electrical stimulation). b, LIVE/DEAD staining of fibroblast cells (Scale bar $=100 \mu \mathrm{m})$. An obvious increase in cell confluency in the experimental group was observed. c, Absorbance ratio of cells in $\mathrm{CP} / \mathrm{electrical}$ stimulation group to those in the control group at $450 \mathrm{~nm}$ by CCK- 8 assay. $\mathbf{d}$, e, ELISA assay analyses of EGF (d) and TGF- $\beta$ (e) expression concentration from cell culture supernatant as the function of cell treatment time, with (red) or without (control, blue) CP/electrical stimulation. Two-way ANOVA was used for statistical analysis. ${ }^{*} \mathrm{p}<0.05$, $* \mathrm{p}<0.01$. The shaded regions in panels c-e represent standard deviations calculated from three measurements. $\mathbf{f}, \mathbf{g}$, an illustrative schematics and digital photograph of the $\mathrm{CP}$ electrodes for heart pacing and porcine heart attached with $\mathrm{CP}$ electrodes. h, Comparison of the threshold voltage for heart pacing by using different electrodes. i, ECG signal recorded during pacing, and schematics of the ECG lead placement. 
that having the $\mathrm{CP}_{18 \mathrm{M}}$ on a hydrogel ionic conductor could effectively boost the current pass through this region, with respect to the regions not covered by CP (Supplementary Figs. S48 and S49). Furthermore, when the AC power frequency decreased down to $1 \mathrm{~Hz}$, the current density of the section without CP hydrogel reduced seriously by more than $50 \%$, reaching only $5 \%$ of the current relative to that of the section with 4 pieces of $\mathrm{CP}$ hydrogel $\left(\sim 21 \mathrm{~mA} \mathrm{~cm}{ }^{-2}\right)$ (Fig. 4g and 4h, Supplementary Fig. 50). Additionally, all the sections with $\mathrm{CP}_{18 \mathrm{M}}$ showed a sinusoidal-shaped waveform, but irregular shapes were observed for sections in absence of $\mathrm{CP}_{18 \mathrm{M}}$, as the result of severe interference of electrochemical reactions occurring within the metal/electrolyte interfaces, consistent with the previous impedance results. This ionic conductor is also stretchable (Fig. 4i, Supplementary Fig. S51). The $\mathrm{CP}_{18 \mathrm{M}}$ positions in the simulation result (the interfaces were assumed bonded without detachment) showed a good agreement with the experiment, indicating the device integrity could be maintained even after stretching, owing to the excellent adhesiveness of the PAAc hydrogel. Overall, ionic conductors with spatial-resolved current densities can be fabricated through patterning $\mathrm{CP}$ hydrogel on the surface of ionic conductors, providing a new ionotronic model for multiplexed sensors and spatial-resolved neural stimulators with soft, stretchable, and partially transparent properties.

\section{Biocompatibility, benefits in cell proliferation and heart pacing}

Considering the promising applications of soft ionic-conductor-based devices in bioelectronics and the good biocompatibility of $\mathrm{CP}$ hydrogel as reported previously, the biological function of CP hydrogel was studied by taking advantage of its excellent conductivity. Electrical stimulations have been studied and employed as a strategy for therapeutic function in clinical usage recently, including wound healing treatment, neuron regeneration, and infracted myocardium repair ${ }^{33}$. To assess the therapeutic function of our CP hydrogel, fibroblast cells, which played an essential role in the wound healing process, were incubated on our semi-transparent $\mathrm{CP}$ hydrogel membrane, and their behavior with electrical stimulation applied was in vitro studied (Fig. 5a). Compared with the control group, the fibroblast cells in the group of culturing with CP hydrogel membrane with electric fields had much higher cell confluency (Fig. 5b). Moreover, the LIVE/DEAD staining results showed that most of the cells were alive (green), revealing the good biocompatibility of our CP hydrogel membrane. Noteworthy, many cells stayed round on PEDOT:PSS hydrogel after 48 $\mathrm{h}$ culturing, suggesting that our CP hydrogel membrane was non-adherent. Such a merit was 
vital for clinical usage to develop easy-detachable devices, such as wound dressing and temporary implantable scaffolds. ${ }^{48,49}$ Furthermore, the cell viability and proliferation after applying electric stimulation were measured by a CCK- 8 assay. The cell viability reached the highest percentage after 48 has $146.8 \% \pm 4.27 \%(n=3)$. The positive correlation of cell viability ratio with time represented in Fig. $5 \mathrm{c}$ demonstrated the electric field function in facilitating fibroblast cell proliferation.

To further understand the detailed mechanism and the role of electric stimulation through $\mathrm{CP}$ hydrogel in promoting cell proliferation, the alternation of growth factor expression in experimental groups was explored by collecting cell culturing supernatant at $4,12,24$, and 48 $\mathrm{h}$, respectively. As a result, the epidermal growth factor (EGF) and transforming growth factorbeta (TGF- $\beta$ ) (Fig. 5d and e), two important growth factors involved in regulating wound healing, showed elevated expression in cells cultured with $\mathrm{CP}$ hydrogel with statistical significance ( $\mathrm{p}=0.0099$ and 0.0268 respectively). Such an enhanced expression suggested that the increased cell proliferation induced by $\mathrm{CP}$ hydrogel could be mapped to growth factors and cellular metabolism pathways, providing a new approach to regulating cell proliferation, assisting wound treatment, and even potentially serving as scaffolds for promoting tissue regeneration by $\mathrm{CP}$ hydrogels.

Ultimately, to prove the practical applicability and advantages of the CP hydrogel in bioelectronics as a better live tissue-electronic interface, a pair of CP electrodes with exposed areas of $\sim 4 \mathrm{~mm}^{2}$ were fabricated as epicardial electrodes to pace an euthanized porcine heart (Fig. 5f-h). If using commercial metal electrodes and porous $\mathrm{Pt} / \mathrm{C}$ electrodes, for comparison, the pacing voltage thresholds for $\mathrm{Au}$ and $\mathrm{Pt} / \mathrm{C}$ electrodes were $1.6 \mathrm{~V}$ and $1.0 \mathrm{~V}$ respectively. By contrast, the $\mathrm{CP}$ electrode could stimulate the heart response at a much lower voltage of $0.4 \mathrm{~V}$, due to its strong electron injection ability. Such a significant stimulating power reduction for $60-75 \%$ presented the great promise of $\mathrm{CP}$ electrodes for a pacemaker operating with ultra-low energy consumption. The successful pacing was further validated through the recording of electrocardiogram traces (Fig. 5i).

\section{Conclusion and prospect}

505 This work introduced a general strategy of preparing and utilizing ultrastrong, highly conductive and capacitive CP hydrogel electrodes, to solve electron-ion transduction issues,

507 including signal loss and distortion and poor voltage tolerance, which are suffered by nearly 508 all electronics when interfacing with biological tissues or ionic conductors. Attributed to the 
509

510

511

512

513

514

515

516

517

518

519

520

521

522

523

524

525

526

527

528

529

530

531

532

533

534

535

536

537

538

539

540

541

highly-oriented nanoporous structures in the $\mathrm{CP}$ hydrogel realized by a gelation-densification strategy, these transduction issues were successfully remedied, enabling better interfacial stability and long-term operation without significant faradic electrochemical processes. Furthermore, by taking advantage of the magnificent difference between CPIT and CIT, an ionic conductor with current density spatial modulation was for the first time demonstrated, providing a new design strategy of spatial-resolved ionic conductors for new functions of ionotronics (e.g., multipoint stimulation by a single probe). Additionally, the great promotion of biological cell proliferation by electrical stimulation and significantly lower voltage threshold of heart pacing using the $\mathrm{CP}$ epicardial electrodes, presented not only the advantages in bioelectronics applications with direct tissue contact (e.g., tissue healing and neuron regenerations), and also the strong ion-injection ability. Solving this critical issue makes a step forward for ionotronics and bioelectronics to better bridge the electronic realm with the biological world expectedly, with great promise in transforming human-machine interactions, energy harvesting, sensing, etc.

Besides the hydrogel-based ionotronics, this CPIT strategy is also expected to be applicable for other subsets of ionic conductors (such as ionic liquids or polyelectrolyte-based ionotronics). The simplicity of this design by using a polymer film electrode allows for combining other functional soft materials, such as bioadhesion. Moreover, considering the high conductivity, easy fabrication, and outstanding performance on reducing the electrode/electrolyte interfacial impedance, the CP hydrogels can be directly employed to replace metal to fabricate various ionotronics for much better performance, such as improving the rectifying performance of ionic-based transistors, the output performance of triboelectric generators, and the signal-noise ratio of neural probes.

\section{Methods}

Surface gelation and patterning of CP. Typically, polydimethylsiloxane (PDMS, Sylgard 184 silicone elastomer, Dow) plates were coated with a layer of $\mathrm{Fe}_{\mathrm{mp}}$ of 1-3 $\mu \mathrm{m}$ (40337 iron powder, spherical, Alfa Aesar) by spray-coating $50 \mathrm{mg} \mathrm{mL}^{-1} \mathrm{Fe}_{\mathrm{mp}}$ isopropanol solution (Supplementary Fig. S3). Subsequently, 6 $\mathrm{mL}$ of pristine PDEOT:PSS suspension (11-13 mg mL $\mathrm{mL}^{-1}$, Celvios PH1000, Heraeus) was dropped onto PDMS substrate $\left(5.4 \times 5.4 \mathrm{~cm}^{2}\right)$ and kept static for $12 \mathrm{~h}$. Finally, CP hydrogel on PDMS was obtained after etching residual $\mathrm{Fe}_{\mathrm{mp}}$ by $0.5 \mathrm{M} \mathrm{H}_{2} \mathrm{SO}_{4}$. CP hydrogel with a low or high loading was also prepared by using the $3.5 \mathrm{~mL}$ or $8.3 \mathrm{~mL}$ of pristine $\mathrm{CP}$ suspension as the precursor.

For the patterning of CP hydrogel, $\mathrm{Fe}_{\mathrm{mp}}$-coated PDMS was attached with a mask (Magic tape, Scotch) with a designed pattern (fabricated by laser cutting (LS-3655, Bosslaser)). $3 \mathrm{mg} \mathrm{mL}^{-1} \mathrm{CP}$ suspension was dropped 
onto the substrate and kept still for 20 min. Finally, CP hydrogel with patterns was obtained after removal of the mask and etching the residual $\mathrm{Fe}_{\mathrm{mp}}$ by $0.5 \mathrm{M} \mathrm{H}_{2} \mathrm{SO}_{4}$.

Preparations of the free-standing CP hydrogel. The as-prepared CP hydrogel on the PDMS substrate was chemically treated by $\mathrm{H}_{2} \mathrm{SO}_{4}$ solution with a gradient concentration from $1 \mathrm{~mol} \mathrm{~L}^{-1}$ to $12 \mathrm{~mol} \mathrm{~L}^{-1}$. Then, the

547 free-standing $\mathrm{CP}_{12 \mathrm{M}}$ was obtained after the dialysis in purified water for 2 days to remove $\mathrm{H}_{2} \mathrm{SO}_{4}$. The $\mathrm{CP}_{18 \mathrm{M}}$ 548 was obtained by further treating free-standing $\mathrm{CP}_{12 \mathrm{M}}$ with concentrated $\mathrm{H}_{2} \mathrm{SO}_{4}$, followed by dialysis for 2 549 days. $\mathrm{CP}_{\mathrm{D} 20 \%}, \mathrm{CP}_{\mathrm{D} 10 \%}$, and $\mathrm{CP}_{\mathrm{D} 5 \%}$ were densified $\mathrm{CP}_{18 \mathrm{M}}$, which were prepared by exchanging water in $\mathrm{CP}_{18 \mathrm{M}}$

550

551

552

553

554

555

556

557

558

559

560

561

562

563

564

565

566

567

568

569

570

571

572 Characterizations of structure: SEM micrographs were taken out on a field emission scanning electron

Preparations of optically transparent $\mathbf{C P}$ hydrogel membrane. $1.5 \mathrm{mg} \mathrm{mL}^{-1} \mathrm{CP}$ suspension was dropped onto a $\mathrm{Fe}_{\mathrm{mp}}$-coated PDMS substrate and kept static for $30 \mathrm{~s}$ to $5 \mathrm{~min}$, followed by chemical treatment by $\mathrm{H}_{2} \mathrm{SO}_{4}$ solution with gradient concentration from $1 \mathrm{~mol} \mathrm{~L}^{-1}$ to $12 \mathrm{~mol} \mathrm{~L}^{-1}$. Finally, transparent $\mathrm{CP}$ hydrogel membranes supported by a PDMS substrate was obtained after dialysis for $12 \mathrm{~h}$. The optically transparent CP hydrogel are beneficial for observation, and thus were used for the cell culturing studies.

Fabrication of ionic conductors. Typically, without particular notice, a PVA hydrogel (length, width, and thickness is $4 \mathrm{~cm}, 1.1 \mathrm{~cm}$, and $0.4 \mathrm{~cm}$, respectively) was employed as a matrix and was infiltrated with different electrolytes ( $1 \mathrm{M} \mathrm{H}_{2} \mathrm{SO}_{4}$, or $3,1,0.5$, or $0.154 \mathrm{M} \mathrm{NaCl}$ solution) to fabricate ionic conductor, which was attached with two pieces of Pt plates $\left(0.9 \times 0.9 \mathrm{~cm}^{2}\right)$ for CIT or $\mathrm{CP}_{18 \mathrm{M}}$ hydrogels $\left(0.9 \times 0.9 \mathrm{~cm}^{2}\right)$ for CPIT at its two ends. Finally, the ionic conductor was connected to external circuits through Pt leads for tests. Noteworthy, the PVA hydrogel had shrinkage in the size when $\mathrm{NaCl}$ solution with high concentration was infiltrated (the size of PVA changed into $2.7 \times 0.95 \times 0.35 \mathrm{~cm}^{3}$ ). Besides, for the generality study of CPITs, PVA hydrogel strip with the same width $(1.1 \mathrm{~cm})$ and thickness $(0.4 \mathrm{~cm})$ but with different lengths $(6.4 \mathrm{~cm}$ and $14 \mathrm{~cm}$ ) were also employed as matrix and was infiltrated with $3 \mathrm{M} \mathrm{NaCl}$ electrolytes to fabricate ionic conductor. Hydrogels from different polymers (PAAc, poly(N-isopropylacrylamide), polyacrylamide) were also prepared, infiltrated with $3 \mathrm{M} \mathrm{NaCl}$ electrolyte, and finally cut into a similar size of $\sim 2.7 \times 0.95 \times 0.35$ $\mathrm{cm}^{3}$ to fabricate ionic conductors.

573 microscope (Supra 40VP, ZEISS). XPS was obtained using an X-ray photoelectron spectrometer (Axis Ultra,

574 Kratos) with an exciting line of Al Ka. Raman spectra were recorded by a confocal Raman microscope upon 575 the laser of $633 \mathrm{~nm}$ (inVia Inspect, Renishaw). Ultraviolet-visible (UV-vis) spectra were recorded by UV576 vis spectrometer (UV-3101PC, Shimadzu).

577 
578 Electrical conduction measurement. For $\mathrm{CP}_{12 \mathrm{M}}, \mathrm{CP}_{18 \mathrm{M}}$, and its densified counterparts, conductivity was 579 measured by using a four-point probe head with tungsten carbide electrodes (C4S probe, Cascade 580 Corporation) coupled with a digital multimeter (4200 digital multimeters, Keithley). The CP hydrogel was 581 cut into a square shape of $3 \times 3 \mathrm{~cm}^{2}$, and its conductivity $(\sigma)$ was calculated by Eq. 3 .

$$
\sigma=\frac{\mathrm{I}}{4.53 \times \mathrm{V} \times \mathrm{T}}
$$

583

584

585

586

587

588

589

590

591

592

593

594

595

596

597

598

599

600

601

602

603

604

605

606

607

608

609

610

611

, where I, V, and T are current, voltage, and thickness of the CP hydrogel, respectively.

For transparent CP hydrogels supported by PDMS substrates, the sheet resistance $\left(\mathrm{R}_{\mathrm{s}}\right)$ was measured by using the CP hydrogel with a rectangular shape of $3 \times 1 \mathrm{~cm}^{2}$, through the AC impedance method in a twoelectrode configuration on an electrochemical working station (CHI660E, $\mathrm{CH}$ Instrument) with the amplitude of $5 \mathrm{mV}$. To avoid the dehydration of $\mathrm{CP}$ hydrogel, purified water was dropped onto the $\mathrm{CP}$ hydrogel. The sheet resistances of CP hydrogel were calculated by Eq. 4, and were fitted against the optical transmittance at $418 \mathrm{~nm}$ by Eq. 5 .

$$
R_{S}(\omega)=\frac{|\mathrm{Z}(\omega)| \times \mathrm{W}}{\mathrm{L}}
$$

, where $|\mathrm{Z}(\omega)|$ is the modulus of impedance, $\mathrm{L}$ is the distance between two electrodes, $\mathrm{W}$ is the width of CP hydrogel.

$$
\mathrm{T}=\left(1+\frac{\mathrm{Z}_{0}}{2 \mathrm{R}_{\mathrm{s}}} \frac{\sigma_{\mathrm{op}}}{\sigma_{\mathrm{dc}}}\right)^{-2}
$$

, where $T$ is the transmittance, $Z_{0}$ is the impedance of free space $(377 \Omega)$, Rs is the sheet resistance, $\sigma_{\mathrm{op}}$ and $\sigma_{\mathrm{dc}}$ are the optical and electrical conductivities respectively. According to the fitting results, $\sigma_{\mathrm{op}} / \sigma_{\mathrm{dc}}$ is calculated to be $\sim 20$.

Mechanical measurements. PEDOT:PSS hydrogels were cut into a rectangular shape with a width of $1 \mathrm{~cm}^{2}$, and its tensile strain-stress curves were recorded on a dynamic mechanical analyzer (DMA850, TA instruments) in a tensile mode at the strain rate of $100 \%$ strain per minute (gauge length $=3 \mathrm{~cm}$ ). Before the tests, the hydrogels were spray-coated with enough water to avoid the dehydration of the PEDOT:PSS hydrogel.

Electrochemical characterizations in a three-electrode system. Three-electrode system was employed to study the intrinsic electrochemical properties of the PEDOT:PSS hydrogel through an electrochemical working station (CHI660E, CH Instrument). Free-standing PEDOT:PSS hydrogel $\left(0.9 \times 0.9 \mathrm{~cm}^{2}\right), \mathrm{Pd} / \mathrm{Au}$ plate $\left(3 \times 1 \mathrm{~cm}^{2}\right)$, SCE were employed as working, counter, and reference electrodes, respectively. $0.1 \mathrm{~mol}$ $\mathrm{L}^{-1} \mathrm{H}_{2} \mathrm{SO}_{4}$ or $\mathrm{NaCl}$ of different concentrations $\left(3,1,0.5\right.$, or $\left.0.154 \mathrm{~mol} \mathrm{~L}^{-1}\right)$ was employed as an electrolyte. In CV and GCD measurements, the electrochemical windows were controlled to be $0-0.8 \mathrm{~V}\left(\mathrm{vs} . \mathrm{Hg} / \mathrm{Hg}_{2} \mathrm{Cl}_{2}\right.$ ) for all these electrolytes except $3 \mathrm{~mol} \mathrm{~L}^{-1} \mathrm{NaCl}$ electrolyte (0-0.7 V). EIS spectra were obtained at an initial potential of $0 \mathrm{~V}$ (vs. SCE) with $5 \mathrm{mV}$ amplitude in the frequency range of $10^{5}-0.025 \mathrm{~Hz}$. The areal 
612 capacitance $\left(\mathrm{C}_{\mathrm{A}}\right)$, specific volumetric capacitance $\left(\mathrm{C}_{\mathrm{V}}\right)$, IR drop $\left(\mathrm{V}_{\mathrm{IR}}\right)$, and coulombic efficiency $(\mathrm{CE})$ were

613 calculated by Eqs. 6-9 respectively.

$$
\mathrm{C}_{\mathrm{A}}=\frac{\mathrm{I} \times \mathrm{t}_{\text {cathodic }}}{\mathrm{A} \times \mathrm{E}_{\text {high }}}
$$

$$
\mathrm{C}_{\mathrm{A}}=\frac{\mathrm{I} \times \mathrm{t}_{\text {cathodic }}}{\mathrm{V} \times \mathrm{E}_{\mathrm{high}}}
$$

616

$$
\mathrm{V}_{\mathrm{IR}}=\mathrm{E}_{\text {high }}-\mathrm{E}_{\text {ini }}
$$

$$
\mathrm{CE}=\frac{\mathrm{t}_{\text {cathodic }}}{\mathrm{t}_{\text {anodic }}}
$$

, where $\mathrm{I}$ is cathodic or anodic current, $\mathrm{t}_{\text {cathodic }}$ and $\mathrm{t}_{\text {anodic }}$ are cathodic and anodic time respectively, $\mathrm{E}_{\max }$ and $\mathrm{A}$ and $\mathrm{v}$ are the area and volume of the working electrode.

621

The areal real capacitance $\left(C^{\prime}\right)$ and resistor-capacitor time constant $\left(\tau_{\mathrm{RC}}\right)$ derived from EIS spectra were calculated by Eqs. 10 and 11.

$$
\begin{aligned}
& C^{\prime}(\omega)=\frac{-Z^{\prime \prime}(\omega)}{\mathrm{A} \times \omega \times|\mathrm{Z}(\omega)|^{2}} \\
& t_{R C}=\mathrm{Z}^{\prime}(\omega) \times \frac{-Z^{\prime \prime}(\omega)}{\mathrm{A} \times \omega \times|\mathrm{Z}(\omega)|^{2}}
\end{aligned}
$$

625

$|Z(\omega)|$ is the modulus of impedance, $Z^{\prime}(\omega), Z^{\prime}{ }^{\prime}(\omega)$ are real and imaginary parts of impedance, $A$ is areal of working electrodes, and $\omega$ is the angular frequency.

Impedance test and analysis of ionic conductors. Except for particular notice, the impedances of CIT and CPIT were recorded by an electrochemical working station (CHI660E, CH Instrument) at the voltage of $0 \mathrm{~V}$ with an amplitude of $5 \mathrm{mV}$ from $10^{5}$ to $0.02 \mathrm{~Hz}$. The CIT and CPIT were connected to the electrochemical working station by Pt wire. The diffusion resistances were obtained from EIS spectra according to the electrochemical model (See Supplementary Discussion Section 4 for details).

634 Electric measurement of ionotronics loaded with resistors or LED. For ionotronics loaded with a resistor,

635 a CIT or CPIT was fabricated as ionotronic devices by employing a PVA hydrogel $\left(2.7 \times 0.95 \times 0.35 \mathrm{~cm}^{3}\right)$ as 636 polymer matrixes, $3 \mathrm{M} \mathrm{NaCl}$ solution as electrolytes, and two $\mathrm{CP}_{18 \mathrm{M}}$ hydrogels $\left(0.9 \times 0.9 \mathrm{~cm}^{2}\right.$, areal capacitance $\sim 110 \mathrm{~F} \mathrm{~cm}^{-2}$ at $\left.0.62 \mathrm{~mA} \mathrm{~cm}^{-2}\right)$ for CPITs or Pt plate $\left(0.9 \times 0.4 \mathrm{~cm}^{2}\right)$ for CITs as electrodes. Upon an AC power, the voltages across the resistors were calculated from the measured currents by the Ohm's 639 Law.

640 For ionotronics loaded with LEDs, A CIT or CPIT was fabricated by employing a PVA hydrogel (length: 7 cm, width: $1.2 \mathrm{~cm}$, thickness $0.4 \mathrm{~cm}$ ) as polymer matrixes, $3 \mathrm{M} \mathrm{NaCl}$ as electrolytes, and two $\mathrm{CP}_{18 \mathrm{M}}$ hydrogels $\left(0.9 \times 0.4 \mathrm{~cm}^{2}\right.$, areal capacitance $\sim 110 \mathrm{~F} \mathrm{~cm}^{-2}$ at $\left.0.62 \mathrm{~mA} \mathrm{~cm}{ }^{-2}\right)$ or Pt plate $\left(0.9 \times 0.4 \mathrm{~cm}^{2}\right)$ as

643 electrodes. Then, Two LEDs with antiparallel polarity were connected to an electrochemical working station 644 (as AC power source and current meter) through a CIT or CPIT, which was then powered by an AC voltage 
with a triangular waveform (voltage window: -3 to $3 \mathrm{~V}$ ). The voltage across the LEDs and the ionic conductor were recorded by a digital multimeter (4200 digital multimeters, Keithley) in real-time.

648 Ionic conductors with spatial-resolved current densities. Adhensive PAAc-hydrogel-based ionic 649 conductor was prepared after $8 \mathrm{~g}$ acrylic acid, $15 \mathrm{mg}$ bis, $30 \mathrm{mg}$ VA-044 were added into $32 \mathrm{~mL}$ of water, 650 kept still at $60^{\circ} \mathrm{C}$ for $30 \mathrm{~min}$, and infiltrated with $3 \mathrm{M} \mathrm{NaCl}$ solutions. Then, $\mathrm{CP}_{18 \mathrm{M}}$ hydrogels were cut into 651 a rectangular shape of $\sim 0.2 \times 0.2 \mathrm{~cm}^{2}$ and attached to the surface of PAAc hydrogel directly. For the current density test, the ionic conductor was divided into 9 domains $\left(9.3 \times 9.3 \mathrm{~mm}^{2}\right)$, and all these domains acted as top electrodes connected in parallel through Pt plates, while another $\mathrm{CP}_{18 \mathrm{M}}$ hydrogel adhered on the other side of PAAc hydrogel acted as the bottom electrodes. Top and bottom electrodes were connected to an electrochemical working station as an AC power source and applied by an input AC voltage ( $\mathrm{V}=0.848$ sin $(2 \pi f \mathrm{t})$ where $f=1$ or $10 \mathrm{~Hz})$. The currents passing through each domain were recorded in real-time by a digital multimeter (4200 digital multimeters, Keithley).

658

Mechanical Simulation of the ionotronics with spatial-resolved current densities. The simulation was done using SolidWorks ${ }^{\circledR}$. The mechanical property of the PAAc hydrogel was measured using DMA 850 (Texas Instruments) and was used as the input for the simulation. The mechanical property of the $\mathrm{CP}_{18 \mathrm{M}}$ hydrogel previously mentioned in this work was used. The bonding between $\mathrm{CP}_{18 \mathrm{M}}$ and PAAc hydrogel was set as bonded to represent a non-slip attachment. Two additional blocks with extremely high modulus are introduced to the model and set to bond with the PAAc, to ensure that the two ends of the hydrogel are stretched homogeneously. To ensure an acceptable simulation time and satisfactory accuracy, we set the mesh to be denser around the PEDOT and sparser at other areas. The non-linear simulation was run with the final state of the PAAc to have the same length as in the experiment.

Cell culture and electric stimulation. NIH 3T3 fibroblast cells were cultured in Dulbecco's modified Eagle's medium (Thermofisher Scientific) supplemented with $10 \%$ bovine serum (Thermofisher Scientific) in PDMS molds with the size of $5 \times 1 \mathrm{~mm}^{2}$. For control groups, cells were directly cultured on Petri dishes. For experimental groups, cells were cultured directly on CP-coated PDMS connected to Arbitrary Function Generator (AFG1000, Tektronix Inc.) by gold electrodes. The parameter for electric stimulation (verified with an oscilloscope) was set up as $2 \mathrm{~Hz}, 1.2 \mathrm{~V} / \mathrm{mm}$ with $1 \mathrm{~ms}$ pulse width according to the previous studies. ${ }^{45}$ All devices for cell culture were sterilized by UV light for $20 \mathrm{~min}$.

676

677 Cell viability test. To assess cell viability after being treated with or without PEDOT:PSS for $48 \mathrm{~h}$, cells 678 were stained by using LIVE/DEAD cell staining kit (Thermofisher Scientific), washed with DPBS twice, and then evaluated by microscope (Zeiss Axio observer, Zeiss). To test cell proliferation, after 10\% CCK-8 was added in each well and incubated for $2 \mathrm{~h}$ at $37^{\circ} \mathrm{C}$, the absorbance at $450 \mathrm{~nm}$ was measured by a plate reader ( $\mathrm{n}=3$ ) (SpectraMax M5, Molecular Devices). The growth factors secreted by fibroblast cells were 
tested by EGF and TGF- $\beta$ ELISA assay kit (Thermofisher Scientific). Briefly, the supernatant of cell culture mediums was collected for each well at 4, 12, 24, $48 \mathrm{~h}$ after seeding of cells and electric stimulation, and were analyzed by a microplate reader (SpectraMax M5, Molecular Devices).

685

Ex vivo ventricular pacing. A postmortem study was performed in a male Yucatan miniature pig (S \& S Farms, Ranchita, Calif). All animal studies were approved by the UCLA Office of Animal Research in compliance with the UCLA IACUC protocols. The porcine epicardium was exposed through thoracotomy conducted by an experienced veterinarian from the UCLA Department of Animal and Laboratory Medicine. Then, the CP electrodes with an exposed areal of $4 \mathrm{~mm}^{2}$ were attached onto the epicardium of the right ventricles. Immediately after euthanasia, external pacing with an amplitude of $2 \mathrm{~V}$, pulse width of $1 \mathrm{~ms}$ at the rate of 60 beat per minutes was initiated according to previous literature ${ }^{34}$, while a simultaneous electrocardiogram (ECG) was recorded for monitoring cardiac rhythm and assessing pacemaker effectiveness.

Data availability. The data that support the plots within this paper and other findings of this study are available from the corresponding authors on request.

699

700

701

702

703

704

\section{Acknowledgments}

X.H. acknowledges NSF CAREER award 1724526, AFOSR awards FA9550-17-1-0311, FA9550-18-

705

706

707

708

709 1-0449 and FA9550-20-1-0344, and ONR awards N000141712117 and N00014-18-1-2314.

710

\section{Additional information}

712 Supplementary information is available for this paper.

713

\section{Competing interests}

715 The authors declare no competing financial interests. 
1. $\quad$ Yang, C. \& Suo, Z. Hydrogel ionotronics. Nat. Rev. Mater. 3, 125-142 (2018).

720 2. Kim, D.H. et al. Materials for multifunctional balloon catheters with capabilities in cardiac

3. Wise, K.D., Anderson, D.J., Hetke, J.F., Kipke, D.R. \& Najafi, K. Wireless Implantable Microsystems: High-Density Electronic Interfaces to the Nervous System. Proceedings of the IEEE 92, 76-97 (2004).

4. Boehler, C., Carli, S., Fadiga, L., Stieglitz, T. \& Asplund, M. Tutorial: guidelines for standardized performance tests for electrodes intended for neural interfaces and bioelectronics. Nat. Protoc. 15, 3557-3578 (2020).

5. Song, E., Li, J., Won, S.M., Bai, W. \& Rogers, J.A. Materials for flexible bioelectronic systems as chronic neural interfaces. Nat. Mater. 19, 590-603 (2020).

6. Climbing-inspired twining electrodes using shape memory for peripheral nerve stimulation and recording. Sci. Adv. 5, eaaw1066 (2019).

7. Fang, Y. et al. Micelle-enabled self-assembly of porous and monolithic carbon membranes for bioelectronic interfaces. Nat. Nanotechnol. 16, 206-213 (2021).

8. Chun, J. et al. Boosted output performance of triboelectric nanogenerator via electric double layer effect. Nat. Commun. 7, 12985 (2016).

9. Schroeder, T.B.H. et al. An electric-eel-inspired soft power source from stacked hydrogels. Nature 552, 214-218 (2017).

10. Zou, Y. et al. A bionic stretchable nanogenerator for underwater sensing and energy harvesting. Nat. Commun. 10, 2695 (2019).

11. Kim, B. et al. Robust High Thermoelectric Harvesting Under a Self-Humidifying Bilayer of Metal Organic Framework and Hydrogel Layer. Adv. Funct. Mater. 29, 1807549 (2019).

12. Tybrandt, K., Forchheimer, R. \& Berggren, M. Logic gates based on ion transistors. Nat. Commun. 3, 871 (2012).

13. Tybrandt, K., Larsson, K.C., Richter-Dahlfors, A. \& Berggren, M. Ion bipolar junction transistors. Proc. Natl. Acad. Sci. U.S.A. 107, 9929-9932 (2010).

14. Yang, C.H., Chen, B., Zhou, J., Chen, Y.M. \& Suo, Z. Electroluminescence of Giant Stretchability. Adv. Mater. 28, 4480-4484 (2016).

15. Liu, X., Liu, J., Lin, S. \& Zhao, X. Hydrogel machines. Mater. Today 36, 102-104 (2020).

16. Li, T.F. et al. Fast-moving soft electronic fish. Sci. Adv. 3, e1602045 (2017).

17. Zhu, B., Gong, S. \& Cheng, W. Softening gold for elastronics. Chem. Soc. Rev. 48, 1668-1711 (2019).

18. Son, D. et al. Multifunctional wearable devices for diagnosis and therapy of movement disorders. Nat. Nanotechnol. 9, 397-404 (2014).

19. Keplinger, C. et al. Stretchable, Transparent, Ionic Conductors. Science 341, 984 (2013).

20. Liu, J. et al. Intrinsically stretchable electrode array enabled in vivo electrophysiological mapping of atrial fibrillation at cellular resolution. Proc. Natl. Acad. Sci. U.S.A. 117, 1476914778 (2020).

21. Yu, Y., Nyein, H.Y.Y., Gao, W. \& Javey, A. Flexible Electrochemical Bioelectronics: The Rise of In Situ Bioanalysis. Adv. Mater. 32, e1902083 (2020).

22. Hong, G. \& Lieber, C.M. Novel electrode technologies for neural recordings. Nat. Rev. Neurosci. 20, 330-345 (2019).

23. Li, Z. et al. Tuning the interlayer spacing of graphene laminate films for efficient pore utilization towards compact capacitive energy storage. Nat. Energy 5, 160-168 (2020).

24. Yang, X., Cheng, C., Wang, Y., Qiu, L. \& Li, D. Liquid-Mediated Dense Integration of Graphene Materials for Compact Capacitive Energy Storage. Science 341, 534-537 (2013).

25. Sheberla, D. et al. Conductive MOF electrodes for stable supercapacitors with high areal capacitance. Nat. Mater. 16, 220-224 (2017). 
26. Yao, B. et al. Ultrahigh-Conductivity Polymer Hydrogels with Arbitrary Structures. Adv. Mater. 29, 1700974 (2017).

27. Feig, V.R. et al. An Electrochemical Gelation Method for Patterning Conductive PEDOT:PSS Hydrogels. Adv. Mater., e1902869 (2019).

28. Lu, B. et al. Pure PEDOT:PSS hydrogels. Nat. Commun. 10, 1043 (2019).

29. Zhang, S.M. et al. Room-Temperature-Formed PEDOT:PSS Hydrogels Enable Injectable, Soft, and Healable Organic Bioelectronics. Adv. Mater. 32, 7 (2020).

30. Govindarajan, A.V., Je, M., Park, W.T. \& Achyuta, A.K.H. in MEMS for Biomedical Applications. (eds. S. Bhansali \& A. Vasudev) 361-395 (Woodhead Publishing, 2012).

31. Chen, G. et al. Strain- and Strain-Rate-Invariant Conductance in a Stretchable and Compressible 3D Conducting Polymer Foam. Matter 1, 205-218 (2019).

32. Xia, Y.J., Sun, K. \& Ouyang, J.Y. Solution-processed metallic conducting polymer films as transparent electrode of optoelectronic devices. Adv. Mater. 24, 2436-2440 (2012).

33. Nunes, S.S. et al. Biowire: a platform for maturation of human pluripotent stem cell-derived cardiomyocytes. Nat. Methods 10, 781-787 (2013).

34. Abiri, P. et al. Inductively powered wireless pacing via a miniature pacemaker and remote stimulation control system. Sci Rep. 7, 6180 (2017). 


\section{Supplementary Files}

This is a list of supplementary files associated with this preprint. Click to download.

- SIfinal.docx 